\title{
SNAP $_{c}$ : a core promoter factor with a built-in DNA-binding damper that is deactivated by the Oct-1 POU domain
}

\author{
Vivek Mittal, Beicong Ma, and Nouria Hernandez ${ }^{1}$ \\ Howard Hughes Medical Institute and Cold Spring Harbor Laboratory, Cold Spring Harbor, New York 11724 USA
}

\begin{abstract}
snRNA gene transcription is activated in part by recruitment of $\operatorname{SNAP}_{c}$ to the core promoter through protein-protein contacts with the POU domain of the enhancer-binding factor Oct-1. We show that a

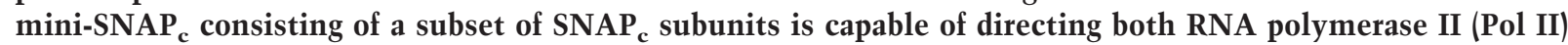
and Pol III snRNA gene transcription. Mini-SNAP ${ }_{c}$ cannot be recruited by Oct-1, but binds as efficiently to the promoter as SNAP ${ }_{c}$ together with Oct-1 and directs activated RNA Pol III transcription. Thus, SNAP represses its own binding to DNA, and repression is relieved by interactions with the Oct-1 POU domain that promote cooperative binding. We have shown previously that TBP also represses its own binding, and in that case repression is relieved by cooperative interactions with $\operatorname{SNAP}_{c}$. This may represent a general mechanism to ensure that core promoter-binding factors, which have strikingly slow off-rates, are recruited specifically to promoter sequences rather than to cryptic-binding sites in the genome.
\end{abstract}

[Key Words: snRNA genes; $\mathrm{SNAP}_{\mathrm{c}}$ PSE; Oct-1 POU; TBP; mini-SNAP ; transcription activation]

Received May 13, 1999; revised version accepted June 7, 1999.

The core elements of RNA polymerase I (Pol I) and Pol II promoters are recognized by the multisubunit factors SL1 and TFIID, respectively, and the core elements of RNA Pol III promoters are recognized by TFIIIA and TFIIIC or just TFIIIC. These factors function to direct the assembly of the correct transcription initiation complex in a regulated manner. The size and complexity of these factors provide them with the flexibility required to perform their task, in particular the flexibility to interact with the large number of proteins imposed by a combinatorial mechanism of transcription regulation. For example, at least in vitro, basal RNA Pol II transcription from a TATA box containing RNA Pol II mRNA promoter can be achieved with just the TATA box-binding protein TBP, but response to activators requires the TBPassociated factors (TAFs) that together with TBP constitute the TFIID complex (for review, see Tansey and Herr 1997). Partial TFIID complexes can only respond to the subsets of activators with which they are capable of associating, suggesting that direct protein-protein contacts between activators and TFIID are important for the activation process (Chen et al. 1994). Thus, the various TFIID subunits provide TFIID with the flexibility to interact with the vast number of transcription factor combinations that regulate expression from mRNA promoters.

${ }^{1}$ Corresponding author.

E-MAIL hernande@cshl.org; FAX (516) 367-6801.
The basal transcription factor $\mathrm{SNAP}_{\mathrm{c}}$ (snRNA activating protein complex) provides a unique model system to dissect the roles of the various subunits of a core promoter-binding factor. $\mathrm{SNAP}_{\mathrm{c}}$, also called PTF, binds specifically to a core promoter element, referred to as the proximal sequence element, or PSE, which is present in both the RNA Pol II and Pol III snRNA promoters (Murphy et al. 1992; Sadowski et al. 1993). In the RNA Pol II snRNA promoters, the PSE is the sole element required to direct basal levels of transcription in vitro, and thus $\mathrm{SNAP}_{\mathrm{c}}$ on its own nucleate the assembly of an RNA Pol II transcription initiation complex. In the RNA Pol III snRNA promoters, the PSE functions in concert with a TATA box located downstream to direct basal RNA Pol III transcription, and thus SNAP $_{c}$ together with TBP bound to the TATA box nucleates the assembly of an RNA Pol III transcription initiation complex (for review, see Henry et al. 1998a). On RNA Pol III snRNA promoters, $\mathrm{SNAP}_{\mathrm{c}}$ and TBP bind cooperatively to their respective targets, and this effect depends on the nonconserved amino-terminal domain of TBP (Mittal and Hernandez 1997). $\mathrm{SNAP}_{\mathrm{c}}$ is well defined and consists of five subunits, SNAP190, SNAP50/PTFß, SNAP45/PTFס, SNAP43/PTF $\gamma$, and SNAP19 (for review, see Henry et al. 1998a), and recombinant $\mathrm{SNAP}_{\mathrm{c}}$ is functional for both RNA Pol II and Pol III snRNA gene transcription (Henry et al. 1998b).

$\mathrm{SNAP}_{\mathrm{c}}$ constitutes a direct target for the transcriptional activator Oct-1, which binds to an octamer sequence present in the enhancer, or distal sequence ele- 
ment (DSE), of snRNA promoters (for review, see Henry et al. 1988a). Oct-1, a POU domain protein (Herr et al. 1988), binds cooperatively with $\mathrm{SNAP}_{\mathrm{c}}$ to the DNA (Murphy et al. 1992; Mittal et al. 1996). This effect results from a direct protein-protein interaction involving

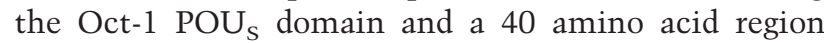
within the carboxy terminal region of SNAP190, and promotes transcription activation (Mittal et al. 1996; Ford et al. 1998). The Oct-1 POU domain, then, contributes to transcription activation of snRNA promoters by recruiting $\mathrm{SNAP}_{\mathrm{c}}$ to the PSE. Thus, $\mathrm{SNAP}_{\mathrm{c}}$ is involved in a number of well-defined functions, that is, specific binding to the PSE, nucleation of RNA Pol II and Pol III transcription initiation complexes, cooperative binding with TBP, and cooperative binding with Oct-1 POU.

Here, we have defined the role of the various $\mathrm{SNAP}_{\mathrm{c}}$ subunits in these processes by assembling partial $\mathrm{SNAP}_{\mathrm{C}} \mathrm{S}$ in vitro. We find that a mini-SNAP $\mathrm{C}_{\mathrm{c}}$ consisting of the amino-terminal third of SNAP190, SNAP43, and SNAP50 is capable of binding specifically to the PSE. This complex can also direct both basal RNA Pol II and Pol III snRNA gene transcription in vitro, and it binds cooperatively with TBP on RNA polymerase III snRNA promoters. Remarkably, mini-SNAP ${ }_{c}$ does not bind cooperatively with the Oct-1 POU domain to DNA but its binding is as efficient as that of $\mathrm{SNAP}_{\mathrm{c}}$ recruited by the Oct-1 POU domain, and it directs activated levels of transcription from an RNA Pol III snRNA promoter. Together, these data indicate that the carboxy-terminal region of SNAP190 acts as a damper of DNA binding that is deactivated by protein-protein contacts with the Oct-1 POU domain, which promote cooperative binding. Such a mechanism probably ensures recruitment of $\mathrm{SNAP}_{\mathrm{c}}$ to the correct promoter sequences within the genome.

\section{Results}

The amino-terminal third of SNAP190 mediates association with SNAP19 and SNAP43, whereas the carboxy-terminal region mediates association with SNAP45

SNAP $_{c}$ is a five-subunit complex consisting of SNAP190, SNAP50, SNAP45, SNAP43, and SNAP19 (Henry et al. 1998b). To understand the structural and functional aspects of the complex, it is important to determine its architecture. Our previous coimmunoprecipitation studies have shown that SNAP190 does not associate with SNAP50 but associates directly with each SNAP19 and SNAP45, and that it associates efficiently with SNAP43 in the presence, but not in the absence, of SNAP19 (Henry et al. 1998a; Wong et al. 1998). To determine which regions of SNAP190 are responsible for these associations, we tested the abilities of in vitro translated full-length HA-tagged SNAP190 and the HA-tagged SNAP190 truncations shown in Figure 1A to associate with (1) SNAP45, (2) SNAP19, (3) SNAP43, and (4) SNAP19 and SNAP43 together, in a coimmunoprecipitation assay performed with anti-HA tag monoclonal an- tibodies (mAb) (Niman et al. 1983). The results of these experiments are summarized in Figure $1 \mathrm{~A}$; as an example of the data, coimmunoprecipitations with the HASNAP190(800-1469) truncation are shown in Figure 1B.

With full-length HA-SNAP190, we observed, consistent with our previous results (Henry et al. 1998a; Wong et al. 1998), efficient coimmunoprecipitation of SNAP45 as well as SNAP19, and efficient coimmunoprecipitation of SNAP43 in the presence, but not in the absence, of SNAP19 (Fig. 1A; data not shown). With the HASNAP190(1-505) truncation, which contains the aminoterminal sequences of SNAP190 to amino acid 505, we also observed efficient coimmunoprecipitation of SNAP19 and efficient coimmunoprecipitation of SNAP43 in the presence, but not in the absence, of SNAP19. It is worth noting, however, that we could observe a low level of SNAP43 coimmunoprecipitate with both HA-SNAP190 and the HA-SNAP190(1-505) truncation even in the absence of SNAP19 (see Fig. 1A), which was not detected in our previous experiments (Henry et al. 1998b). We did not test whether the HA-SNAP190(1-505) truncation coimmunoprecipitated with SNAP45, but the larger truncation SNAP190(1-900) did not coimmunoprecipitate with SNAP45. Thus, SNAP19 and SNAP43, but not SNAP45, associate with the amino-terminal part of SNAP190.

When we tested the HA-SNAP190(800-1469) truncation, which contains the carboxy-terminal half of the protein, we obtained different results, as shown in Figure 1B. With this SNAP190 derivative, we did not observe coimmunoprecipitation of SNAP19, SNAP43 together with SNAP19, or SNAP43 alone (lanes 8-10), but we did observe coimmunoprecipitation of SNAP45 (lane 7). As expected, the anti-HA antibody did not cross-react detectably with untagged SNAP45 (lane 11). Thus, SNAP19 and SNAP43 associate only with the aminoterminal part of SNAP190, and SNAP45 associates only with the carboxy-terminal part of SNAP190.

Figure $1 \mathrm{C}$ shows a schematic of $\mathrm{SNAP}_{\mathrm{c}}$ indicating the protein-protein interactions between $\mathrm{SNAP}_{\mathrm{c}}$ subunits robust enough to be detected in coimmunoprecipitation assays of in vitro-translated proteins. As shown above, SNAP45 interacts directly with the carboxy-terminal half of SNAP190, whereas SNAP19 interacts directly with the amino-terminal third of SNAP190. SNAP43 joins the complex through interactions with both SNAP19 and SNAP190. SNAP50 can only be coimmunoprecipitated with SNAP43, suggesting that it joins the complex through direct interaction with SNAP43 (Henry et al. 1998a). The stoichiometry of the individual subunits in $\mathrm{SNAP}_{\mathrm{c}}$ is not known.

The Rc and Rd repeats of SNAP190 are essential for efficient binding of $S N A P_{c}$ to the PSE

As shown in Figure 2A, the largest subunit of $\mathrm{SNAP}_{\mathrm{c}}$ SNAP190, contains an unusual Myb domain consisting of four repeats designated $\mathrm{Ra}, \mathrm{Rb}, \mathrm{Rc}$, and $\mathrm{Rd}$, preceded by a half repeat designated $\mathrm{Rh}$, rather than the more 
A

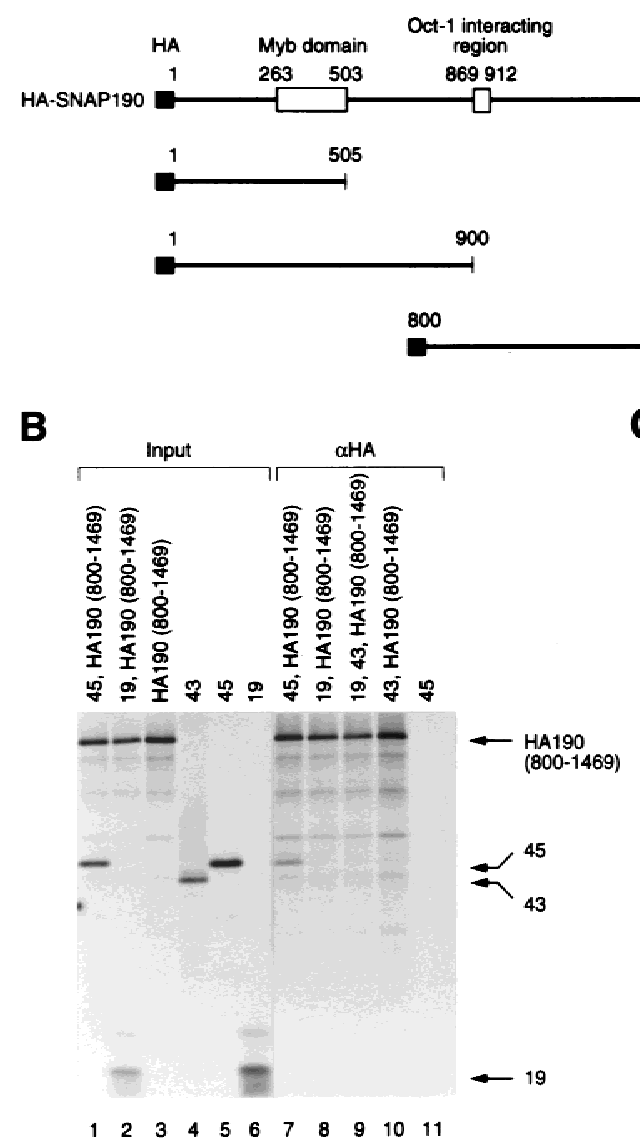

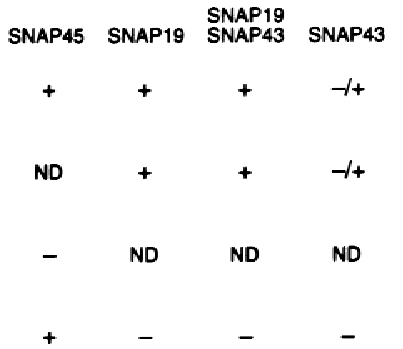

Figure 1. The SNAP190 amino-terminal third mediates association with SNAP19 and SNAP43, whereas the SNAP190 carboxy-terminal half mediates association with SNAP45. (A) Schematic of full-length and truncated HA-tagged SNAP190 derivatives. The coimmunoprecipitation results are summarized at right. (B) The combinations of $\left[{ }^{35} \mathrm{~S}\right]$ methionine-labeled $\mathrm{SNAP}_{\mathrm{c}}$ subunits indicated above the lanes were either cotranslated or translated separately and then coincubated in rabbit reticulocyte lysates. The products were then used for immunoprecipitations with an anti-HA $(\alpha \mathrm{HA})$ antibody, and the immunoprecipitates were fractionated by $15 \%$ SDSPAGE. The radiolabeled proteins were detected by autoradiography. (Lanes 1-6) Ten percent of the input proteins used (either alone or in combinations) for the immunoprecipitations. $(C)$ Architecture of $\mathrm{SNAP}_{\mathrm{c}}$. The stoichiometry of the various $\mathrm{SNAP}_{\mathrm{c}}$ subunits is not known. usual two or three repeats (Wong et al. 1998). We showed before that although full-length SNAP190 and various SNAP190 truncations did not bind DNA, a truncation consisting of just the Rc and Rd repeats bound specifically to the PSE (Wong et al. 1998). However, the role of the various SNAP190 Myb repeats for DNA binding of the entire complex was not determined.

Because the yield of in vitro-translated proteins is too low for binding studies, we turned to the baculovirus expression system to characterize the role of the SNAP190 Myb repeats in binding of SNAP ${ }_{c}$ to the PSE. A functional recombinant $\mathrm{SNAP}_{\mathrm{c}}$ can be assembled by coinfection of insect cells with five recombinant baculoviruses, each expressing one of the $\mathrm{SNAP}_{\mathrm{c}}$ subunits, and purified to near homogeneity by two successive affinity purification steps (Henry et al. 1998b). We coinfected insect cells with recombinant baculoviruses expressing SNAP19, SNAP43, SNAP45, and SNAP50 carrying at its amino terminus an HA tag, and SNAP190 or SNAP190 variants (carrying a histidine tag at their carboxyl terminus). As shown in Figure 2A,

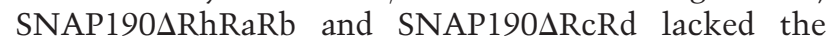
$\mathrm{RhRaRb}$ and $\mathrm{RcRd} \mathrm{Myb}$ repeats, respectively. The resulting complexes were purified first by immunoaffinity with an anti-SNAP190 antibody followed by peptide elution, and second by immunoaffinity with the anti-HA antibody, which selects for the presence of HA-tagged SNAP50. Immunoblot analysis of the purified complexes with a panel of antibodies specific to the various $\mathrm{SNAP}_{c}$ subunits indicated that the SNAP190 deletions did not interfere with complex assembly (data not shown). We used immunoblot quantitation of the SNAP50 subunit, as shown in Figure 2C, to estimate the relative concentrations of the resulting complexes. Equal amounts of wild-type and mutant $\mathrm{SNAP}_{\mathrm{c}} \mathrm{s}$ were then tested for binding to the PSE in an electrophoretic mobility shift assay (EMSA), and the results are shown in Figure 2B.

$\mathrm{SNAP}_{\mathrm{c}}$ containing SNAP190DRhRaRb bound nearly as efficiently to the PSE as wild-type SNAP $_{\mathrm{c}}$ (cf. lanes 5 and 6 with lanes 2 and 3 ), and the binding was specific as it was not observed with a mutant PSE probe (lanes $4,7)$. In contrast, the binding of $\mathrm{SNAP}_{\mathrm{c}}$ containing SNAP190ARcRd was reduced $>90$-fold as compared with wild-type $\operatorname{SNAP}_{\mathrm{c}}$ (cf. lanes 8 and 9 with lanes 2 and 3 ). Nevertheless, some residual binding could be detected, and this residual binding was specific for the PSE (cf. lanes 9 and 10). Together, these results show that the Rc and $\mathrm{Rd}$ repeats are essential for efficient binding of $\mathrm{SNAP}_{\mathrm{c}}$ to the PSE. In their absence, a very low level of residual-specific binding is observed, which may be contributed by other parts of $\mathrm{SNAP}_{\mathrm{c}}$, either within the SNAP190 subunit or in other $\mathrm{SNAP}_{\mathrm{c}}$ subunits. 


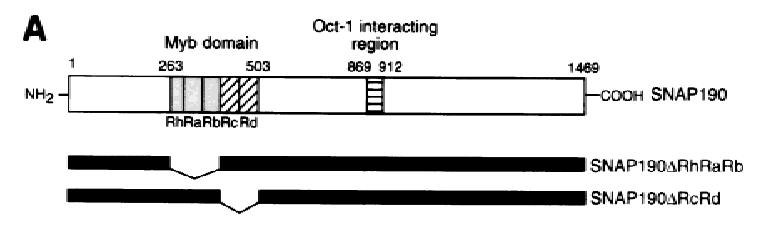

\section{B}

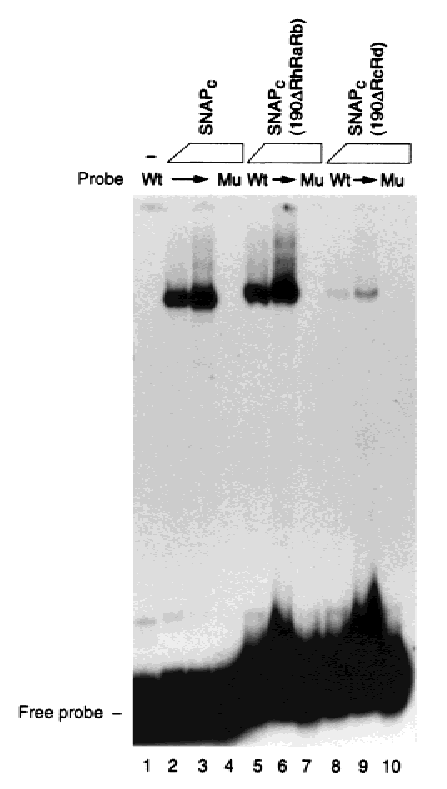

C

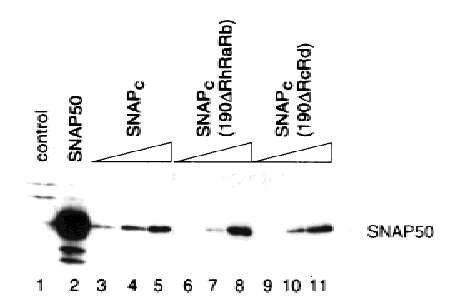

Figure 2. The SNAP190 Rc and $\mathrm{Rd}$ Myb repeats are required for efficient $\mathrm{SNAP}_{\mathrm{c}}$ binding to the PSE. (A) Schematic structure

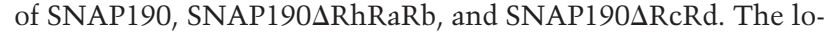
cation of the Myb domain is indicated, as well as the location of the region that interacts with the Oct-1 POU domain (Ford et al. 1998). The regions deleted in SNAP190ARhRaRb and SNAP190 $\Delta$ RcRd are indicated. (B) EMSA performed with SNAP $_{c}$ or the SNAP $_{c}$ derivatives shown above the lanes. The probe contained either a wild-type $(\mathrm{Wt})$ or mutant $(\mathrm{Mu})$ mouse U6 PSE, as indicated above the lanes. We used equivalent amounts of $\mathrm{SNAP}_{\mathrm{c}}$ or derivatives as determined by immunoblot (see Fig. 2C) in the binding reactions, specifically: (lanes 2-4) 1, 2 , and $2 \mu \mathrm{l}$ of $\mathrm{SNAP}_{\mathrm{c}}$ (lanes 5-7), 3, 6, and $6 \mu \mathrm{l}$ of $\mathrm{SNAP}_{\mathrm{c}}$ containing the SNAP190DRhRaRb subunit; (lanes 8-10) 3, 6, and 6

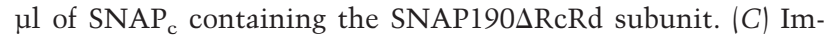
munoblot analysis of $\mathrm{SNAP}_{\mathrm{c}}$ and the $\mathrm{SNAP}_{\mathrm{c}}$ derivatives indicated above the lanes with an anti-SNAP50 antibody. (Lane 1) uninfected Sf9 cell lysate; (lane 2) lysate from Sf9 cells infected with an HA-SNAP50-expressing recombinant baculovirus; (lanes 3-5) 0.17, 0.5, and $1 \mu \mathrm{l}$ of complete $\mathrm{SNAP}_{\mathrm{c} ;}$ and (lanes 6-8)

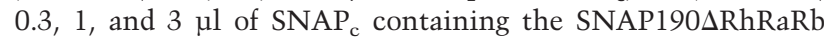
subunit; (lanes 9-11) or the SNAP190ARcRd subunit. SNAP and the $\mathrm{SNAP}_{\mathrm{c}}$ derivatives were purified by sequential antiSNAP190 and anti-HA immunoaffinity steps.
Partial SNAP $C_{C}$ missing SNAP45, SNAP19, or both SNAP19 and SNAP45 are capable of binding to the PSE

Having established the crucial role of SNAP190, and more specifically the Rc and Rd repeats of SNAP190, for specific binding to the PSE, we turned our attention to the other $\mathrm{SNAP}_{\mathrm{c}}$ subunits. We coinfected insect cells with viruses expressing all possible combinations of four of the five SNAP $_{c}$ subunits, as well as one combination of three $\mathrm{SNAP}_{\mathrm{c}}$ subunits. SNAP190 carried a histidine tag at its carboxyl terminus and SNAP50 carried an HA tag at its amino terminus. We devised purification schemes that select for the various subcomplexes and eliminate unassociated subunits. These, as well as the structures of the purified complexes, are summarized in Figure 3A. For each infection, all the $\mathrm{SNAP}_{\mathrm{c}}$ subunits present in the insect cells are shown, with the subunits that assembled into the purified complexes in gray, and those that were not incorporated into the complexes in white. All complexes except those assembled in the absence of SNAP190 were purified first on nickel agarose beads, which select for the presence of the His-tagged SNAP190. The full complex and complexes assembled in the absence of SNAP45, or SNAP19, or both SNAP45 and SNAP19, were further purified on anti-HA mAb beads, which select for the presence of HA-tagged SNAP50. Complexes assembled in the absence of SNAP43 or SNAP50 were not expected to contain SNAP50 (see Fig. 1C and 3A) and were, therefore, not purified further. Complexes assembled in the absence of SNAP190 were purified over anti-HA mAb beads and contained, therefore, HA-tagged SNAP50 and the associated SNAP43. The composition of all complexes was then confirmed by immunoblots with antibodies directed against each of the $\mathrm{SNAP}_{\mathrm{c}}$ subunits, and the results obtained with the anti-SNAP50 antibody are shown in Figure 3C: Complete $\mathrm{SNAP}_{\mathrm{c}}$ and complexes assembled in the absence of SNAP45, SNAP19, both SNAP45 and SNAP19, and SNAP190, all contained SNAP50, whereas complexes assembled in the absence of SNAP43 or SNAP50 did not, as expected.

Equal amounts of purified complete $\mathrm{SNAP}_{\mathrm{c}}$ and partial SNAP $_{\mathrm{c}} \mathrm{s}$ as determined, where possible, by immunoblot quantitation of SNAP50 (see Fig. 3C) or, in the case of partial complexes lacking SNAP50, by quantitation of SNAP190 (data not shown), were tested by EMSA for their ability to bind to the PSE, and the results are shown in Figure 3B. Complete $\mathrm{SNAP}_{\mathrm{c}}$ bound efficiently to the PSE, as expected (lanes 1,2). Partial SNAP $\mathrm{C}_{\mathrm{c}}$ assembled in the absence of SNAP43, SNAP50, or SNAP190 did not display any detectable DNA-binding activity (lanes 9-11). In contrast, a partial $\mathrm{SNAP}_{\mathrm{c}}$ assembled in the absence of SNAP45 formed a weak diffuse complex migrating faster than the complete SNAP $_{\mathrm{c}}$-PSE complex (lanes 3,4). Remarkably, a partial $\mathrm{SNAP}_{\mathrm{c}}$ assembled in the absence of SNAP19 also formed a weak complex migrating at an intermediate position between the complete SNAP $_{\mathrm{c}}-\mathrm{PSE}$ and the $\mathrm{SNAP}_{\mathrm{c}}-45-\mathrm{PSE}$ complexes, consistent with an intermediate molecular weight for this 
A
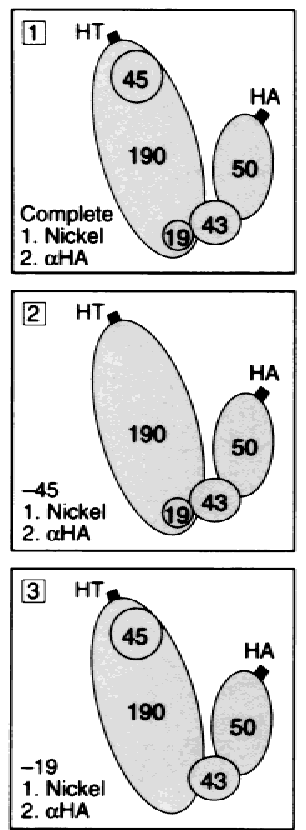
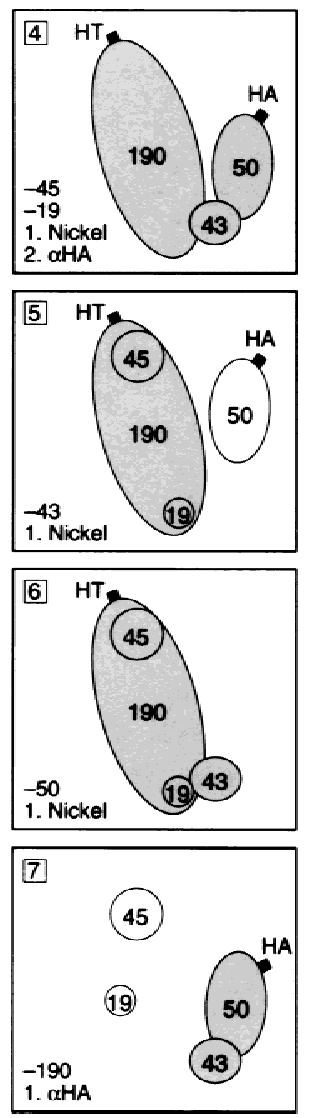

B

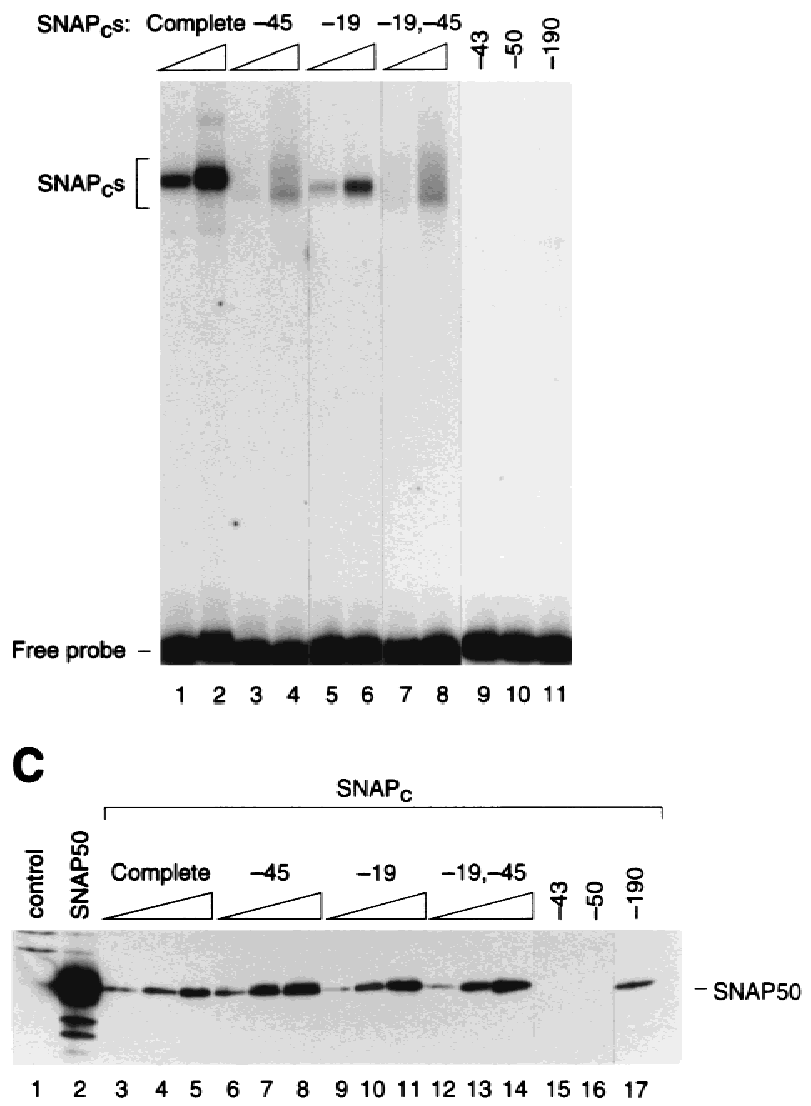

Figure 3. DNA-binding properties of partial SNAP $\mathrm{c}(A)$ Structure of the various partial SNAP $\mathrm{P}_{\mathrm{c}} \mathrm{s}$ used for binding studies. Insect cells were coinfected with four or, in one case, three baculoviruses, each expressing a different $\mathrm{SNAP}_{\mathrm{c}}$ subunit. In each panel, the subunits missing in the infection as well as the steps used to purify the complex are listed (lower left). All subunits present in the insect cells are illustrated at right, with the subunits present in the purified complex shaded. The SNAP190 subunit carries a His tag (HT) at its carboxyl terminus, and the SNAP50 subunit carries an HA tag (HA) at its amino terminus. (B) EMSA performed with either complete $\mathrm{SNAP}_{\mathrm{c}}$ or $\mathrm{SNAP}_{\mathrm{c}} \mathrm{s}$ assembled in the absence of the subunits indicated above the lanes and a probe carrying the high affinity mouse U6 PSE. The structures of the various $\mathrm{SNAP}_{\mathrm{c}} \mathrm{s}$ are illustrated in $A$. We used equivalent amounts of the various complexes as determined by immunoblot (see Fig. 3C) in the binding reactions. (Lanes 1,2) 1 and $2 \mu$ of complete SNAP $_{\text {c }}\left(\right.$ lanes 3,4) 4 and $8 \mu 1$ of SNAP s $^{-}$

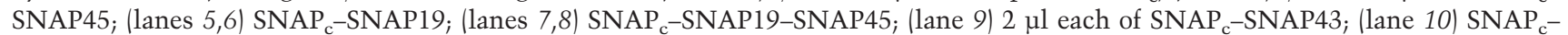

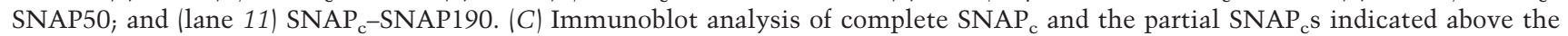
lanes with an anti-SNAP50 antibody. The samples shown are as follows: (Lane 1) uninfected Sf9 cell lysate; (lane 2) lysate from Sf9

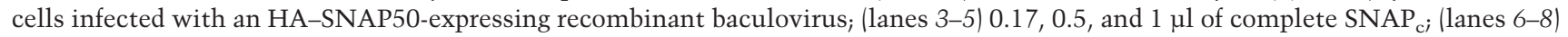
1, 5, and $10 \mu \mathrm{l}$ of $\mathrm{SNAP}_{\mathrm{c}}-\mathrm{SNAP} 45$; (lanes 9-11) SNAP $-\mathrm{SNAP}_{\mathrm{c}}$; ; or (lanes 12-14) SNAP $\mathrm{C}_{\mathrm{c}}-\mathrm{SNAP} 19-\mathrm{SNAP} 45$; and (lane 15) $5 \mu \mathrm{l}$ of $\mathrm{SNAP}_{\mathrm{c}}-\mathrm{SNAP}_{3}$; (lane 16) SNAP - SNAP50; or (lane 17) SNAP - SNAP190.

complex lacking just SNAP19 (lanes 5,6). A complex assembled in the absence of both SNAP45 and SNAP19 formed a weak, diffuse complex migrating, as expected faster than the SNAP ${ }_{c}-45-P S E$ complex (lanes 7,8). None of the protein complexes bound to a probe containing a mutated PSE (data not shown).

Together, these results indicate that complexes missing SNAP19, SNAP45, or both, are still capable of binding, albeit weakly, to the PSE. In contrast, complexes missing SNAP43, SNAP50, or SNAP190 did not bind detectably to the PSE. Thus, consistent with our previous observations that none of the full-length $\mathrm{SNAP}_{\mathrm{c}}$ subunits can bind to DNA on its own, a combination of at least three of the five $\mathrm{SNAP}_{\mathrm{c}}$ subunits, namely
SNAP190, SNAP50, and SNAP43, is necessary to generate detectable DNA-binding ability. Because binding of the complexes assembled in the absence of SNAP19 and/ or SNAP45 was not as efficient as binding of complete SNAP $_{c}$, these data also suggest that SNAP19 and SNAP45 contribute, directly or indirectly, to efficient binding of $\mathrm{SNAP}_{\mathrm{c}}$ to the PSE.

The observation that we could assemble a complex missing SNAP19 in baculovirus-infected cells was surprising because SNAP19 is required for efficient association of SNAP43 and SNAP190 in a coimmunoprecipitation assay of in vitro-translated proteins (Fig. 1; Henry et al. 1998b). We therefore checked, and could confirm, the subunit composition of the complex assembled in the 
absence of SNAP19 by antibody supershift experiments (data not shown). These results suggest that at high SNAP $_{c}$ subunit concentration, as is likely to be the case in baculovirus-infected cells, a $\operatorname{SNAP}_{c}$ missing just SNAP19 can be assembled. As described above, we do observe a low level of association between in-vitro translated SNAP43 and SNAP190 or the amino-terminal third of SNAP190, even in the absence of SNAP19. It is also possible, however, that an insect homolog of SNAP19 that is not recognized by our antibodies gets incorporated into these complexes. We do not consider this likely, however, because the $\mathrm{SNAP}_{\mathrm{c}}-\mathrm{SNAP} 19$ complex migrates significantly faster in the EMSA than the $\mathrm{SNAP}_{\mathrm{c}}$ complex, suggesting that it is missing a subunit.

SNAP45 relieves a binding inhibition conferred by the carboxy-terminal region of SNAP190

$\mathrm{SNAP}_{\mathrm{c}} \mathrm{s}$ assembled in the absence of SNAP45 formed weak and diffuse complexes with the PSE in an EMSA, suggesting that these $\mathrm{SNAP}_{\mathrm{c}} \mathrm{s}$ did not bind efficiently to the PSE. To address the role of SNAP45 in SNAP ${ }_{c}$ binding, we tested the effect of adding increasing amounts of recombinant SNAP45 expressed in Escherichia coli to a partial SNAP $_{c}$ assembled in the absence of SNAP45. As illustrated in Figure 4A, addition of recombinant SNAP45 had no effect on the complete SNAP - PSE complex (cf. lanes 7 and 8). In contrast, however, addition of increasing amounts of SNAP45 to the complex assembled in the absence of SNAP45 resulted in a more intense and more discrete band, which comigrated with the complete SNAP ${ }_{c}-$ PSE complex (lanes 4-6). This result suggests that exogenous SNAP45 produced in E. coli was incorporated into the partial $\operatorname{SNAP}_{\mathrm{c}}$ lacking SNAP45 and stabilized binding to the PSE.

To investigate the mechanism through which SNAP45 stabilizes binding to the PSE, we compared the DNA-binding abilities of equivalent amounts (as determined by quantitation of the SNAP50 subunit by immunoblot; data not shown) of $\mathrm{SNAP}_{\mathrm{c}} \mathrm{s}$ assembled in the absence of SNAP45 and containing either an intact
Figure 4. In the absence of SNAP45, the carboxyterminal region of SNAP190 inhibits binding of SNAP $_{\mathrm{c}}$ to the PSE. (A) EMSA performed with a probe carrying the wild-type mouse U6 PSE and either no proteins (lane 1), increasing amounts of $E$. coli-expressed SNAP45 (lanes 2,3), SNAP $_{\mathrm{c}}-$ SNAP45 alone (lane 4), SNAP - SNAP45 and increasing amounts of E. coli-expressed SNAP45 (lanes 5,6), $\mathrm{SNAP}_{\mathrm{c}}$ alone (lane 7), or $\mathrm{SNAP}_{\mathrm{c}}$ and an amount of $E$. coli expressed SNAP45 equivalent to that used in lane 5. (B) EMSA performed with probes carrying either a wild-type (lanes $1-3,5,6)$ or a mutant (lanes 4,7) mouse U6 PSE and either no protein (lane 1), or equivalent amounts, as determined by immunoblot of the SNAP50 subunit (not shown) of SNAP C $_{\mathrm{c}}$ SNAP45 containing either a fulllength SNAP190 subunit (lanes 2-4) or a SNAP190 subunit missing the carboxy-terminal two thirds of the protein (lanes 5-7). (C) Structure of full-length SNAP190 and SNAP190AC. SNAP190AC is missing amino acids 515-1469 of SNAP190.
A

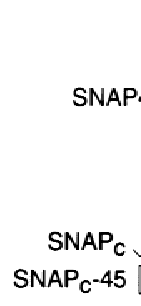
$N_{A P}-45[$
$-$ -45 Complete

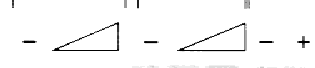

Free probe

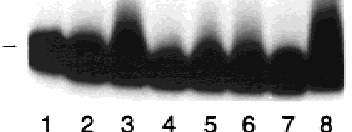

$\begin{array}{llllllll}1 & 2 & 3 & 4 & 5 & 6 & 7 & 8\end{array}$
B

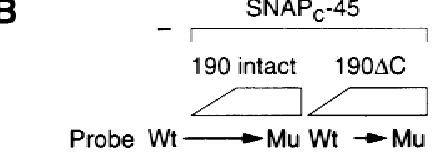

$\mathrm{SNAP}_{\mathrm{C}}-45$

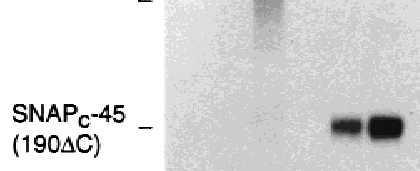

Free probe

C

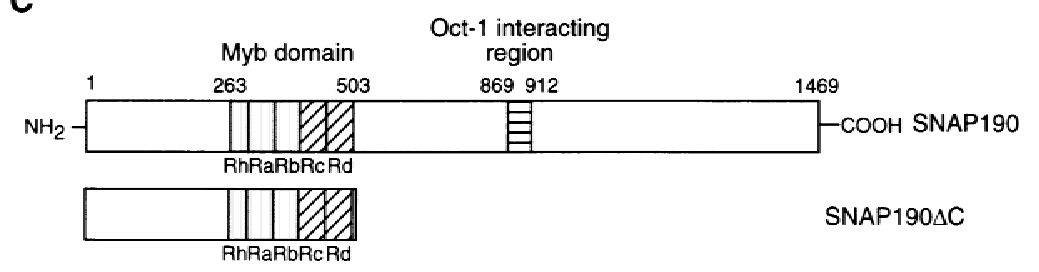


SNAP190 or a SNAP190 missing the carboxy-terminal two-thirds of the protein, as illustrated in Figure 4C. The results are shown in Figure $4 \mathrm{~B}$. As before, a $\mathrm{SNAP}_{\mathrm{c}}$ assembled in the absence of SNAP45 gave rise to a weak and diffuse complex that bound specifically to the PSE (lanes 2-4). In stark contrast, a SNAP ${ }_{c}$ assembled in the absence of SNAP45 and containing only the amino-terminal third of SNAP190 gave rise to a prominent and discrete complex that also bound specifically to the PSE (lanes 5-7). These results show that a complex missing the carboxy-terminal region of SNAP190 and its associated subunit, SNAP45, binds efficiently to the PSE. They are consistent with the idea that in the SNAP ${ }_{c}-$ SNAP45 complex, the carboxy-terminal region of $\mathrm{SNAP}_{\mathrm{c}}$ adopts a flexible conformation that diminishes the ability of the complex to bind to DNA. Addition of SNAP45 engages the carboxy-terminal region of SNAP190 in protein-protein interactions, thus giving it a fixed structure and relieving the inhibition.

A mini-SNAP $P_{c}$ consisting of the amino-terminal third of SNAP190, SNAP43, and SNAP50 is capable

of specific and efficient binding to the PSE

We have shown above that in the absence of the carboxyterminal region of SNAP190, SNAP45 is dispensable for efficient binding to the PSE (Fig. 4), and that a PSE-binding SNAP $_{c}$ can be assembled in the absence of SNAP19 (Fig. 3). In an attempt to define the minimal number of $\mathrm{SNAP}_{\mathrm{c}}$ subunits required for binding, we asked whether we could assemble a mini-SNAP ${ }_{c}$ missing SNAP45, SNAP19, and the carboxy-terminal two-thirds of SNAP190. We coinfected insect cells with recombinant viruses encoding the amino-terminal third of SNAP190 carrying a histidine tag, SNAP43, and HA-tagged SNAP50, and purified the complex first by either immunoaffinity with an anti-SNAP190 antibody or on a nickel agarose column, and second by immunoaffinity with an anti-HA antibody. The subunit composition of the purified complex was determined by fractionation on a SDSpolyacrylamide gel and silver staining, and is shown in Figure 5A, lanes 5 and 6 . Lanes $1-4$ correspond to an autoradiogram of marker lanes in the same gel loaded with a mixture of in vitro-translated SNAP43, HAtagged SNAP50, and an HA-tagged amino-terminal truncation of SNAP190 (lane 1), or each of these in vitrotranslated proteins separately (lanes 2-4). Only three main bands are visible in the mini-SNAP ${ }_{c}$ preparation, even when the gel is overloaded (lane 6), of which the one corresponding to HA-tagged SNAP50 comigrates precisely with the in vitro-translated protein. In contrast, the in vitro-translated SNAP190 truncation and in vitro-translated SNAP43 both migrate slightly more slowly than the proteins expressed in insect cells. This may be due to differences in protein modifications or, in the case of SNAP190, to the different tags and the slightly different lengths of the constructs.

To determine whether mini-SNAP ${ }_{\mathrm{c}}$ was able to bind specifically to the PSE and confirm its subunit composition, we performed the EMSA shown in Figure 5B. To facilitate comparison of the two complexes, the amounts of $\mathrm{SNAP}_{\mathrm{c}}$ and mini-SNAP $\mathrm{C}_{\mathrm{c}}$ were adjusted so as to obtain equivalent amounts of PSE-binding activity. The mobility of the $\mathrm{SNAP}_{\mathrm{c}}-\mathrm{PSE}$ complex was retarded by addition of antibodies directed against peptides from the aminoand carboxy-terminal regions of SNAP190, SNAP50, SNAP45, SNAP43, and SNAP19, as expected (lanes 4-9). In contrast, the mobility of the mini-SNAP ${ }_{c}-P S E$ complex was affected by antibodies directed against a peptide from the amino-terminal region of SNAP190, SNAP50, and SNAP43, but not by antibodies directed against a peptide from the carboxy-terminal region of SNAP190, SNAP45, and SNAP19 (lanes 12-17). Thus, a mini$\mathrm{SNAP}_{\mathrm{c}}$ containing the amino-terminal third of SNAP190, SNAP43, and SNAP50, is capable of binding specifically and efficiently to the PSE.

\section{Mini-SNAP $P_{c}$ binds cooperatively with full-length human TBP on the U6 promoter}

We have shown previously that $\mathrm{SNAP}_{\mathrm{c}}$ and human TBP (hTBP) bind cooperatively to their respective binding sites on the U6 promoter, and that this effect is dependent on the nonconserved amino-terminal domain of hTBP (Mittal and Hernandez 1997). On a probe containing a high affinity PSE such as the mouse U6 PSE, cooperative binding results, in effect, in $\mathrm{SNAP}_{\mathrm{c}}$ recruiting hTBP to the TATA box (Mittal and Hernandez 1997). Which subunits of $\mathrm{SNAP}_{\mathrm{c}}$ are required for this effect has not been determined. Therefore, we were interested in determining whether mini-SNAP ${ }_{\mathrm{c}}$, like $\mathrm{SNAP}_{\mathrm{c}}$ is capable of recruiting hTBP to the U6 TATA box. For this purpose, we used a DNase I footprinting assay, and the results are shown in Figure 6. Mini-SNAP $_{c}$, on its own, efficiently protected the PSE from DNase I digestion (lane 2), and the footprint is very similar to that obtained with $\mathrm{SNAP}_{\mathrm{c}}$ (Mittal and Hernandez 1997). In contrast to the efficient PSE protection obtained with mini-SNAP ${ }_{\mathrm{c}}$ hTBP on its own protected the TATA box only at the higher protein concentration, and the protection was weak (lanes 3,4). Thus, as observed previously (Mittal and Hernandez 1997), under these conditions hTBP did not bind efficiently to the TATA box. When both mini$\mathrm{SNAP}_{\mathrm{c}}$ and hTBP were incubated with the probe, however, TBP was recruited to the TATA box much more efficiently as evidenced by a partial TATA box protection (lanes 5,6) even at the lower concentration of TBP (lane 5). The efficiency of TBP recruitment was similar to that observed with $\mathrm{SNAP}_{\mathrm{c}}$ (data not shown). Thus, like $\mathrm{SNAP}_{\mathrm{c}}$, mini-SNAP ${ }_{\mathrm{c}}$ is capable of recruiting TBP to the TATA box, indicating that the carboxy-terminal two thirds of SNAP190, SNAP45, and SNAP19 are all dispensable for this effect.

\section{Mini-SNAP $P_{c}$ is functional for basal transcription by both RNA Pol II and RNA Pol III}

We have shown before that $\mathrm{SNAP}_{\mathrm{c}}$ is capable of directing transcription from both RNA Pol II and Pol III snRNA 
Mittal et al.
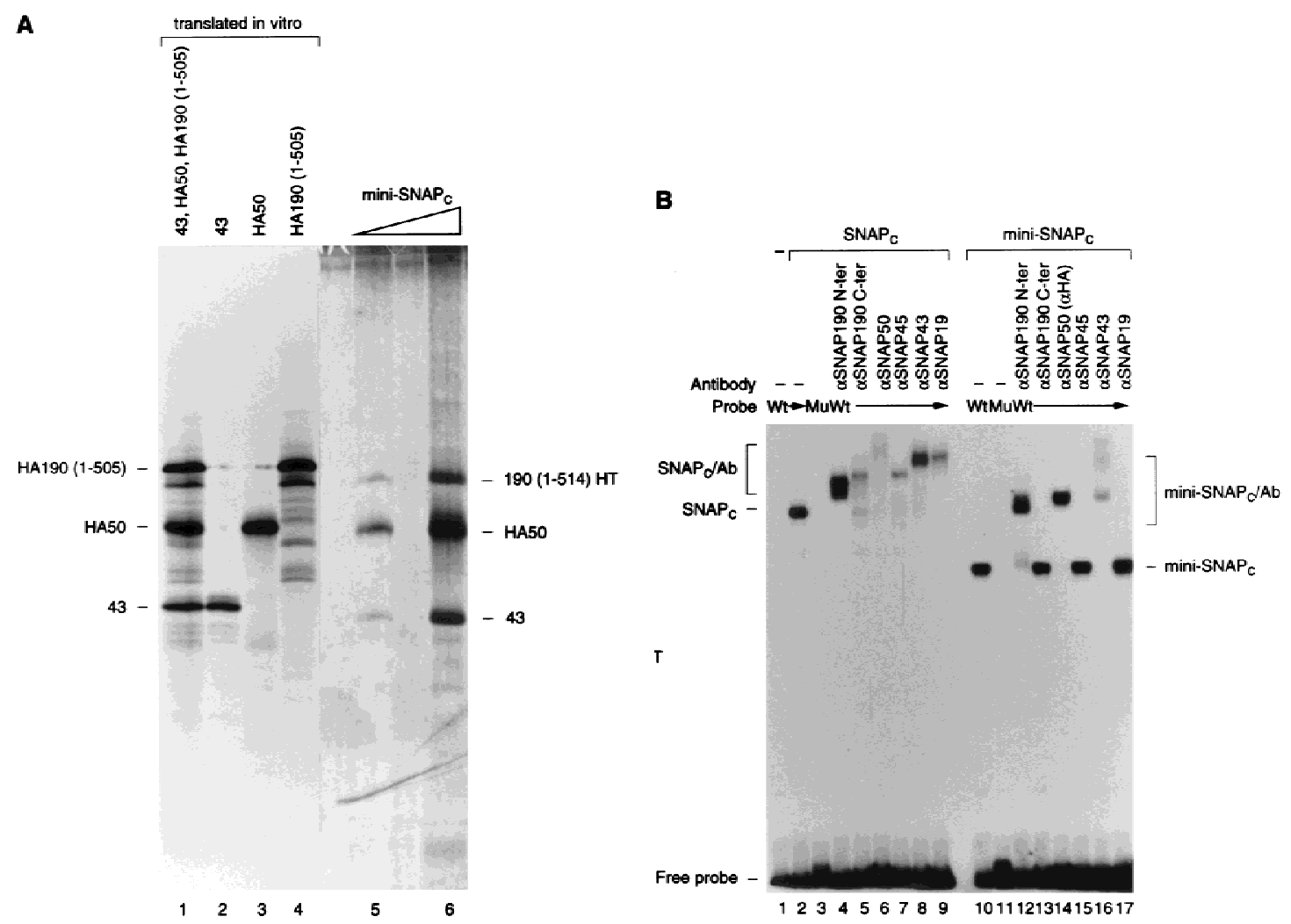

Figure 5. The amino-terminal parts of SNAP190 (amino acids 1-514), SNAP50, and SNAP43 are sufficient for assembly of a miniSNAP $_{\mathrm{c}}$. (A) Composition of mini-SNAP . A mixture of in vitro-translated HA-tagged SNAP190 (1-505), HA-tagged SNAP50, and SNAP43 (lane 1) or SNAP43 (lane 2), HA-tagged SNAP50 (lane 3), and HA-tagged SNAP190 (1-505) (lane 4) alone, was loaded alongside increasing amounts of purified mini-SNAP $($ lanes 5,6). The gel was stained with silver, dried, and photographed (lanes 5,6). The dried gel was then autoradiographed to reveal the location of the radiolabeled in vitro-translated proteins (lanes 1-4). Mini-SNAP ${ }_{c}$ was purified by chromatography over nickel-agarose beads, which selects for the presence of the His-tagged SNAP190 subunit, followed by chromatography over anti-HA antibody beads, which selects for the presence of the HA-tagged SNAP50 subunit. (B) EMSA performed with a probe carrying either the wild-type (lanes 1,2,4-10,12-17) or mutant (lanes 3,11) mouse U6 PSE and either no protein (lane 1) or equivalent DNA-binding units of complete SNAP $_{c}$ (lanes 2-9) or mini-SNAP ${ }_{c}$ (lanes 10-17). (Lanes 4-9,12-13,15-17), anti-peptide polyclonal antibodies directed either against the amino-terminal region of SNAP190 ( $\alpha$ SNAP190 N-ter, CS696), the carboxy-terminal region of SNAP190 ( $\alpha$ SNAP190 C-ter, CS402) or other SNAP ${ }_{c}$ subunits as indicated above the lanes were added to the binding reactions. (Lane 14) The mAb 12CA5 directed against the HA tag on SNAP50 was used. The locations of the SNAP $-\mathrm{PSE}_{\text {, }}$

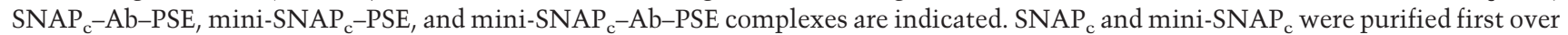
nickel-agarose beads and anti-SNAP190 antibody beads, respectively, which selects for the presence of the His-tagged SNAP190 subunit. Both were then purified over anti-HA antibody beads, which selects for the presence of the HA-tagged SNAP50 subunit.

promoters (Henry et al. 1998b). Because mini-SNAP ${ }_{c}$ is capable of binding specifically to the PSE and recruiting TBP to the TATA box, we tested whether it might be functional for basal transcription. For this purpose, we treated HeLa cell extracts with protein A agarose beads cross-linked to preimmune antibodies or to three antibodies directed against the SNAP19, SNAP45, and SNAP190 subunits of SNAP $_{c}$, respectively. Such a treatment was expected to deplete endogenous $\mathrm{SNAP}_{\mathrm{c}}$ as well as any free SNAP19, SNAP45, or SNAP190 subunit that might be present in the extracts. As shown in Figure 7B, both SNAP45 and SNAP19, which were directly recognized by the antibodies, and SNAP50, which was recognized by virtue of its association with other SNAP $_{c}$ subunits, were efficiently depleted. We then tested the abilities of equivalent amounts of $\operatorname{SNAP}_{c}$ and mini-SNAP ${ }_{c}$ as determined by immunoblot quantitation of both the
SNAP190 and SNAP50 subunits (data not shown), to restore transcription activity to these extracts, and the results are shown in Figure 7A. As templates, we used RNA Pol II and Pol III snRNA promoters that carry the high affinity mouse U6 PSE and lack an octamer sequence upstream, and thus score basal transcription. Extracts depleted with preimmune antibodies directed transcription from both an RNA Pol III and an RNA Pol II snRNA promoter, whereas extracts depleted with the anti-SNAP ${ }_{\mathrm{c}}$ antibodies had little or no activity (cf. lanes 1 and 2). Addition of increasing amounts of recombinant $\mathrm{SNAP}_{\mathrm{c}}$ restored both RNA Pol III and Pol II transcription (lanes 3-5), as observed before (Henry et al. 1998b). Strikingly, addition of equivalent increasing amounts of mini$\mathrm{SNAP}_{\mathrm{c}}$ also restored both RNA Pol III and Pol II transcription (lanes 6-8). In contrast, complexes assembled in the absence of SNAP43 or SNAP50 were inactive 


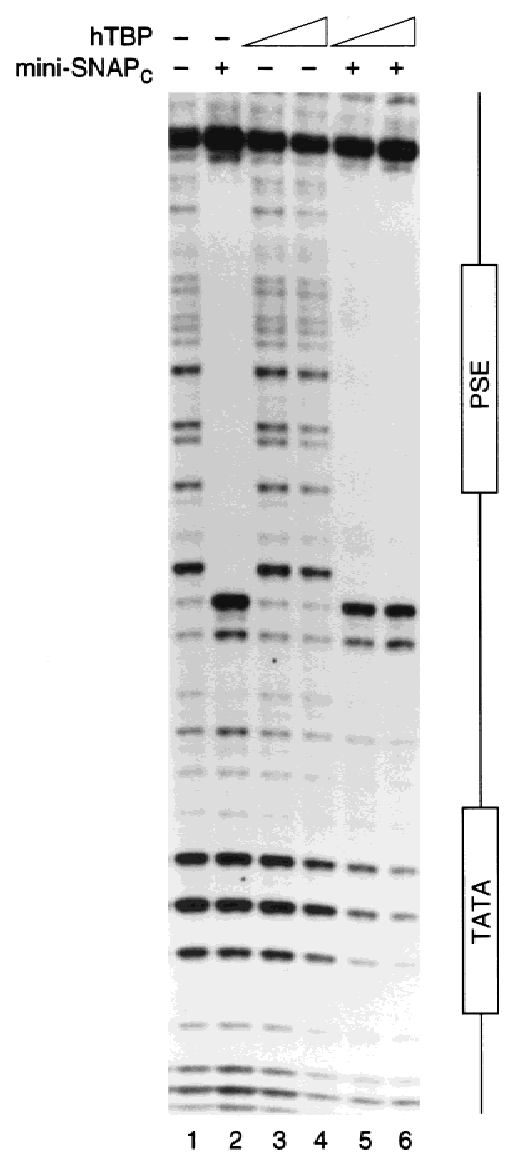

Figure 6. Mini-SNAP can recruit TBP to the TATA box. DNase I footprinting experiment performed with a probe containing the mouse U6 PSE and the human U6 TATA box. The binding reactions contained probe alone (lane 1), mini-SNAP ${ }_{c}$ alone (lane 2), 40 and $80 \mathrm{ng}$ of full-length human TBP (hTBP) alone (lanes 3,4 ), and 40 and $80 \mathrm{ng}$ of hTBP together with mini-SNAP (lanes 5,6 ). The positions of the PSE and the TATA box are indicated at right.

(lanes 9-12). These data indicate that on naked DNA templates, mini-SNAP ${ }_{c}$ is capable of nucleating the assembly of RNA Pol II and Pol III transcription initiation complexes and that SNAP45, SNAP19, and the carboxyterminal two-thirds of SNAP190 are all dispensable for these functions.

\section{Mini-SNAP $P_{c}$ binds efficiently to the PSE independent of the Oct-1 POU domain}

We and others have shown previously that on probes containing both a PSE and an octamer sequence, $\mathrm{SNAP}_{\mathrm{c}}$ and Oct-1 POU bind cooperatively, such that the Oct-1 POU domain in effect recruits SNAP $_{c}$ to the PSE (Murphy et al. 1992; Mittal et al. 1996). Cooperative binding results, at least in part, from a direct protein-protein interaction involving the Oct-1 $\mathrm{POU}_{\mathrm{s}}$ domain as well as a small region within the carboxy-terminal part of SNAP190 (Mittal et al. 1996; Ford et al. 1998). Because mini-SNAP $_{c}$ is missing the entire carboxy-terminal two- thirds of SNAP190, it seemed likely that mini-SNAP would be unable to bind cooperatively with Oct-1 POU. To test this directly, we used a probe containing the H2B octamer, a high affinity Oct-1-binding site, upstream of the human U6 PSE, a weak affinity binding site for SNAP $_{\mathrm{c}}$ (Mittal et al. 1996).

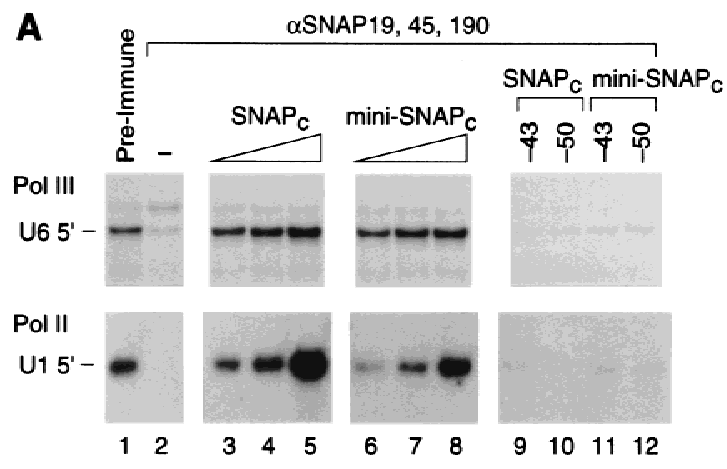

B

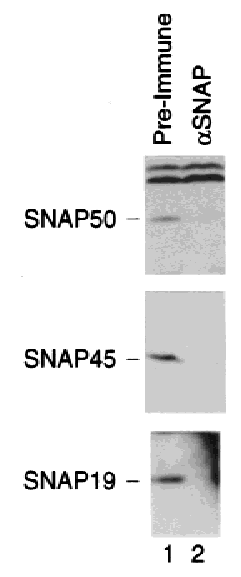

Figure 7. Mini-SNAP ${ }_{c}$ is capable of directing basal transcription by both RNA Pol II and Pol III. (A) HeLa cell extracts were depleted with either rabbit preimmune antibody beads (lane 1), or a mixture of beads carrying anti-peptide antibodies directed against SNAP19, SNAP45, and SNAP190 (lanes 2-12) and tested for their ability to support RNA Pol III transcription from a U6 promoter carrying the mouse U6 PSE (top) and RNA Pol II transcription from a U1 promoter carrying the mouse U6 PSE (bottom; Sadowski et al. 1993). The transcription reactions were supplemented with 1,3 , and $6 \mu l$ of $\operatorname{SNAP}_{c}$ (lanes 3-5), equivalent increasing amounts of mini-SNAP $($ lanes 6-8), and $2 \mu \mathrm{l}$ of SNAP $_{c}$ (lanes 9,10), or mini-SNAP ${ }_{c}$ (lanes 11,12) assembled in the absence of the SNAP43 or SNAP50 subunit, as indicated above the lanes. The purification of $\mathrm{SNAP}_{\mathrm{c}}$ and mini-SNAP ${ }_{\mathrm{c}}$ is summarized in the legend to Fig. $5 \mathrm{~B}$, and the amounts of these complexes were equalized by immunoblot quantitation of the SNAP190 and SNAP50 subunits (not shown). Complexes missing either the SNAP43 or SNAP50 subunits were purified on an anti-SNAP190 antibody column, and their amounts were equalized by immunoblot quantitation of the SNAP190 subunit. (B) Immunoblot analysis of the extracts depleted with either preimmune (lane 1) or anti-SNAP19, anti-SNAP45, and antiSNAP190 beads (lane 2) used in A. The top, middle, and bottom panels were probed with antibodies directed against SNAP50, SNAP45, and SNAP19, respectively. The positions of SNAP50, SNAP45, and SNAP19 are indicated at left. 
As shown in Figure 8A, the binding of $\operatorname{SNAP}_{c}$ alone to the PSE was barely detectable (lanes 1 and 2), but the Oct-1 POU domain bound efficiently to the high affinity octamer (lane 3). When both $\mathrm{SNAP}_{\mathrm{c}}$ and the Oct-1 POU domain were incubated with the probe, a strong complex was obtained (lanes 4,5$)$. We have shown before that this complex contains both the Oct-1 POU domain and SNAP $_{\mathrm{c}}$ (Mittal et al. 1996; Ford and Hernandez 1997; Ford et al. 1998). Unlike $\mathrm{SNAP}_{\mathrm{c}}$, whose binding to the low-affinity human PSE was barely detectable, similar amounts of mini-SNAP ${ }_{c}$ bound very efficiently to the low-affinity human U6 PSE. In fact, the binding of mini-
SNAP $_{c}$ was at least as efficient as that of SNAP $_{c}$ together with Oct-1 POU domain (cf. lanes 6 and 7 with lanes 4 and 5). In the presence of Oct-1 POU, a second, slower migrating complex was obtained, indicating that mini$\mathrm{SNAP}_{\mathrm{c}}$ and Oct-1 POU were capable of co-occupying the probe, but the complex was not significantly enhanced (cf. lanes 8 and 9 with lanes 6 and 7). Thus, the binding of mini-SNAP ${ }_{\mathrm{c}}$ alone could not be enhanced by the Oct-1 POU domain, consistent with our previous results that mapped the Oct-1 POU domain-SNAP ${ }_{c}$ interaction responsible for cooperative binding to amino acids 869912 within the carboxy-terminal two-thirds of SNAP190,

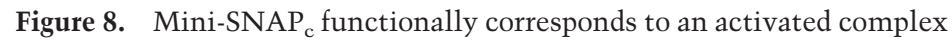
both for PSE-binding and RNA Pol III transcription. (A) EMSA performed with a probe containing the H2B octamer sequence upstream of the human U6 PSE (Mittal et al. 1996) and equivalent increasing amounts of SNAP $_{c}($ lanes $1,2,4,5)$ or mini-SNAP $($ lanes 6-9) either in the absence (lanes 1,2,6,7) or presence (lanes 4,5,8,9) of Oct-1 POU. Oct-1 POU alone was used in lane 3. The SNAP $_{c}-$ PSE complex is barely visible at this exposure, but its location, as well as that of the mini-SNAP ${ }_{c}-P S E$, miniSNAP $_{\mathrm{c}}-\mathrm{POU}-\mathrm{PSE}$, and SNAP ${ }_{\mathrm{c}}-\mathrm{POU}-\mathrm{PSE}$ complexes is shown at left. $(B)$ HeLa cell extracts were depleted with either rabbit preimmune antibody beads (lanes 1,7), or a mixture of beads carrying anti-peptide antibodies directed against SNAP19, SNAP45, and SNAP190 (lanes 2-6, 8-12) and tested for their ability to support RNA Pol III transcription from U6 promoters containing a human U6 PSE and either a wild-type (lanes 1-6) or a mutated (lanes 7-12) H2B octamer sequence (Mittal et al. 1996). The transcription reactions were supplemented with buffer (lanes 1,2,7,8), or 1 and $3 \mu \mathrm{l}$ of $\mathrm{SNAP}_{\mathrm{c}}$ (lanes 3,4,9,10), or equivalent amounts of miniSNAP $_{c}$ (lanes $5,6,11,12$ ). (IC) The position of the signal derived from the internal control RNA added to each sample at the end of the transcription reactions. The signal corresponding to the correct U6 start site (U6 5') is indicated.

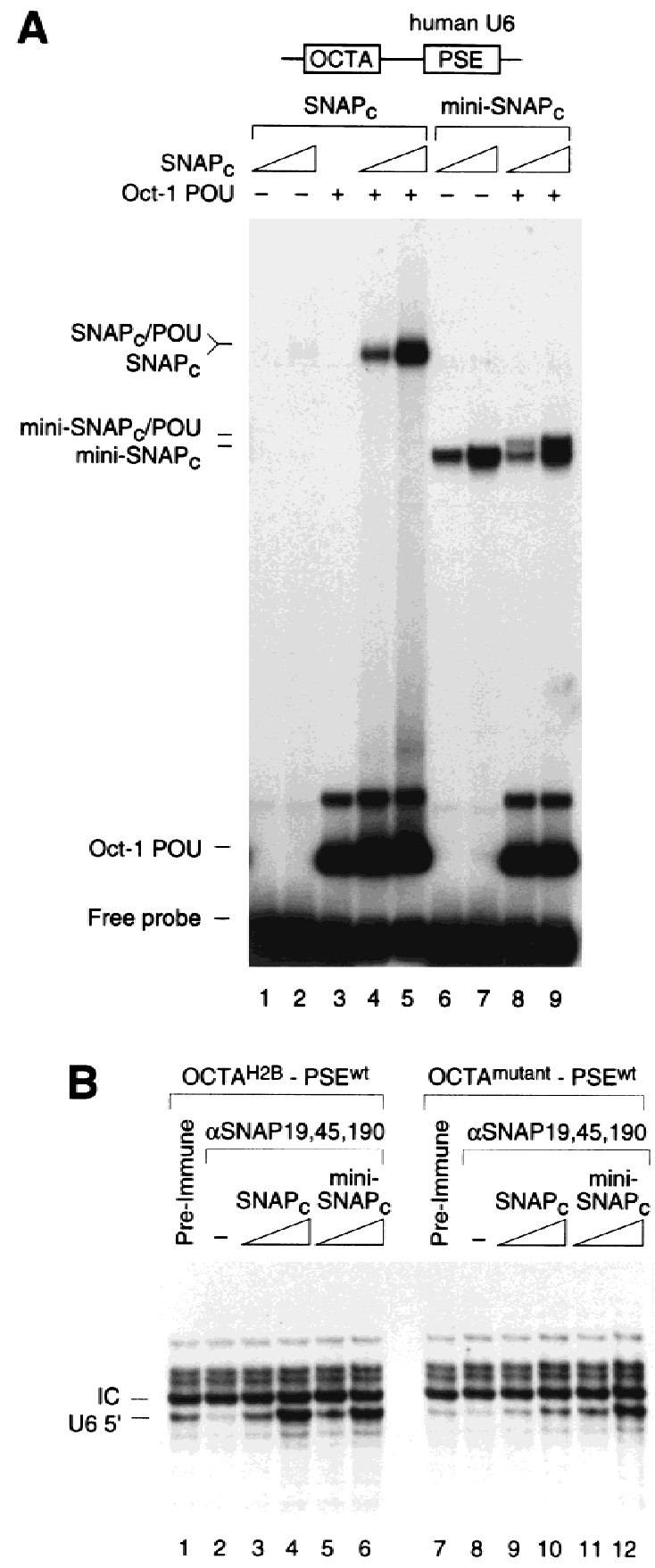


which are absent in mini-SNAP ${ }_{\mathrm{c}}$. The striking result, however, is that mini-SNAP ${ }_{c}$ bound as efficiently to the PSE as SNAP ${ }_{c}$ in the presence of the Oct-1 POU domain. This suggests that the carboxy-terminal domain of SNAP190 down-regulates binding of the complex to the PSE. This down-regulation is counteracted through interaction of the carboxy-terminal domain of SNAP190 with the Oct-1 POU domain.

\section{Mini-SNAP $P_{c}$ is activated for RNA Pol III snRNA gene transcription}

The observation that mini-SNAP bound as efficiently to the PSE as $\mathrm{SNAP}_{\mathrm{c}}$ in the presence of Oct-1 POU prompted us to compare the efficiency of transcription directed by these two complexes, either in the absence (basal transcription) or presence (activated transcription) of an octamer sequence. For this purpose, we depleted a nuclear extract of endogenous SNAP $_{c}$ as above, with antibodies directed against SNAP19, SNAP45, and SNAP190. We then used as a template an RNA Pol III snRNA promoter containing either a wild-type $\left(\mathrm{OCTA}^{\mathrm{H} 2 \mathrm{~B}}-\mathrm{PSE}^{\mathrm{Wt}}\right)$ or a mutated $\left(\mathrm{OCTA}^{\text {mutant}}-\mathrm{PSE}^{\mathrm{Wt}}\right.$ ) octamer site upstream of the low-affinity human U6 PSE. We also included in the transcription reactions an RNA fragment to serve as an internal control (IC) for RNA handling and recovery. As shown in Figure 8B, the template containing a wild-type $\mathrm{H} 2 \mathrm{~B}$ octamer was more active than that containing a mutant octamer sequence (cf. lane 1 with lane 7), indicating that as in vivo (see Lobo and Hernandez 1994), and as observed before (Murphy et al. 1992; Mittal et al. 1996), the octamer activates transcription in this assay. This activation of transcription is dependent on the Oct-1 POU domain (Murphy et al. 1992; Mittal et al. 1996). When the extract was depleted of endogenous $\mathrm{SNAP}_{\mathrm{c}}$, both activated and basal transcription were reduced (lanes 2,8), albeit in the case of basal transcription, this decrease was barely detectable, mainly because the starting level of basal transcription was very low. On addition of increasing amounts of $\mathrm{SNAP}_{\mathrm{c}}$, the template with a wild-type octamer directed higher levels of transcription than the template with a mutated octamer, indicating that in the presence of the octamer sequence, transcription was activated (cf. lanes 3 and 4 with lanes 9 and 10). In sharp contrast, on addition of increasing amounts of mini-SNAP ${ }_{\mathrm{c}}$, both templates directed similar levels of transcription (cf. lanes 5 and 6 with lanes 11 and 12), and strikingly, these levels were as high as those obtained with $\mathrm{SNAP}_{\mathrm{c}}$ on the template containing a wild-type octamer (cf. lanes 5,6,11, and 12 with lanes 3 and 4). Thus, mini-SNAP functionally corresponds to an activated $\mathrm{SNAP}_{\mathrm{c}}$ for RNA Pol III snRNA gene transcription in vitro.

\section{Discussion}

We have dissected the role of the various $\mathrm{SNAP}_{c}$ subunits for a number of $\mathrm{SNAP}_{\mathrm{c}}$ functions including specific binding to the PSE, cooperative binding with TBP and with the Oct-1 POU domain, and basal and activated transcription. Our results show that a mini-SNAP ${ }_{c}$ consisting of SNAP43, SNAP50, and the amino-terminal third of SNAP190 is capable of binding to the PSE, of recruiting TBP to the TATA box, and of directing RNA Pol II and Pol III snRNA gene transcription. Mini-SNAP binds to the PSE as efficiently as $\mathrm{SNAP}_{\mathrm{c}}$ together with the Oct-1 POU domain. Consistent with this observation, mini-SNAP ${ }_{c}$ constitutively directs activated levels of RNA Pol III transcription in vitro.

\section{Architecture of SNAP}

We find that SNAP45 interacts with the carboxy-terminal, and SNAP43 and SNAP19 with the amino-terminal region of SNAP190. We had shown before that SNAP50 interacts only with SNAP43 (Henry et al. 1996, 1998a; Wong et al. 1998). Consistent with these observations, we could assemble partial $\mathrm{SNAP}_{\mathrm{c}} \mathrm{s}$ lacking SNAP45 or SNAP50. More surprisingly, we could also assemble a complex missing just SNAP19, even though in our previous coimmunoprecipitation experiments with in vitro translated proteins, SNAP19 was required for association of SNAP43 and SNAP190 (Henry et al. 1998b). However, we show here that we can detect a weak association of SNAP43 and the amino-terminal region of SNAP190 even in the absence of SNAP19, suggesting that at higher protein concentrations than can be achieved in cotranslations, SNAP43 is able to associate reasonably efficiently with SNAP190 even in the absence of SNAP19. The SNAP $_{\mathrm{c}}-\mathrm{SNAP} 19$ complex may be less stable than $\mathrm{SNAP}_{\mathrm{c}}$, because it displayed reduced binding to the PSE relative to $\mathrm{SNAP}_{\mathrm{c}}$. Alternatively, the reduced binding may reflect a direct role of SNAP19 in contacting the DNA.

The $\mathrm{SNAP}_{\mathrm{c}}$ complex missing just the SNAP45 subunit bound much less efficiently than SNAP $_{c}$ to the PSE, and in an EMSA, the resulting protein-DNA complex was strikingly diffuse. We do not think that this reflects dissociation of the protein-DNA complex during electrophoresis, because this would result in a diffuse signal migrating faster than the expected position of the SNAP $_{\mathrm{c}}-45-\mathrm{SNAP}-\mathrm{PSE}$ complex. Instead, much of the diffuse signal migrated more slowly, suggesting a heterogenous population of SNAP $_{c}-45-S N A P-P S E$ complexes. Because SNAP45 interacts with the carboxy-terminal region of SNAP190, this in turn suggests that in the absence of SNAP45, the carboxy-terminal region of SNAP190 assumes more than one conformation, thereby generating a heterogenous population of $\mathrm{SNAP}_{\mathrm{c}}$ s. Some of these complexes may not be able to bind DNA, thus the overall weaker binding observed with the $\mathrm{SNAP}_{\mathrm{c}^{-}}$ SNAP45 as compared with SNAP $_{c}$. Addition of exogenous SNAP45 probably results in incorporation of SNAP45 into many of the $\mathrm{SNAP}_{\mathrm{c}}-\mathrm{SNAP} 45$ complexes, stabilization of the SNAP190 carboxy-terminal domain conformation, and thus conversion of many of the complexes formerly unable to bind DNA into complexes capable of binding. Cleavage of the carboxy-terminal two 
thirds of SNAP190, which bypasses the requirement for SNAP45 altogether, also resulted in an increase and tightening of the SNAP $_{\mathrm{c}}-\mathrm{PSE}$ complex.

\section{Requirements for binding of $S N A P_{c}$ to the PSE}

The Myb domains of the c-Myb, A-Myb, and B-Myb proteins consist of three repeats called the R1, R2, and R3 repeats, of which the $\mathrm{R} 2$ and $\mathrm{R} 3$ repeats are sufficient for DNA binding (Nomura et al. 1988; Luscher and Eisenman 1990). SNAP190 has an unusual Myb domain consisting of four and a half repeats. We have been able to assemble mutant $\mathrm{SNAP}_{\mathrm{c}} \mathrm{s}$ lacking either the $\mathrm{RhRaRb}$ or the RcRd SNAP190 repeats. In both cases, the complex could be assembled, indicating that the Myb repeats do not mediate essential protein-protein interactions with other $\mathrm{SNAP}_{\mathrm{c}}$ subunits. However, only the complex missing the $\mathrm{RhRaRb}$ repeats was still able to bind efficiently to the PSE. The complex missing the RcRd repeats bound with much reduced efficiency. This is consistent with our previous observations that (1) a small truncation of SNAP190 consisting of just the RcRd repeats could bind to the PSE, and (2) of the four SNAP190 repeats, the $\mathrm{Rc}$ and $\mathrm{Rd}$ repeats are the most similar to the $\mathrm{R} 2$ repeat with $38 \%$ identities each, and the $\mathrm{Rd}$ repeat is the most similar to the R3 repeat with $30 \%$ identities (Wong et al. 1998). Together, these results emphasize the crucial role of the Rc and Rd repeats for $\mathrm{SNAP}_{\mathrm{c}} \mathrm{DNA}$ binding.

It is likely, however, that parts of $\mathrm{SNAP}_{\mathrm{c}}$ other than the SNAP190 Rc and Rd repeats also contribute to DNA binding. Although a small SNAP190 truncation consisting of just the Rc and $\mathrm{Rd}$ repeats was able to bind to the PSE, we have been unable to demonstrate binding by any longer SNAP190 truncation (Wong et al. 1998). When using either full-length SNAP190 or the amino-terminal 514 amino acids of SNAP190, we find that the smallest complex that still binds DNA contains SNAP43 and SNAP50 in addition to SNAP190. This suggests that SNAP50 and/or SNAP43 confer to SNAP190, and more specifically to the amino-terminal region of SNAP190, a structure compatible with DNA binding. In addition, because SNAP50 can be cross-linked to the PSE (Henry et al. 1996), it may provide additional contacts with the DNA.

\section{A mini-SNAP $P_{c}$ active for both basal RNA Pol II and Pol III snRNA gene transcription}

The smallest complex we have generated so far that is still capable of binding to the PSE contains the aminoterminal region of SNAP190, SNAP43, and SNAP50. Because this minicomplex is assembled in insect cells, it is difficult to exclude that it contains an insect cell subunit. We consider this unlikely, however, because silver staining of purified mini-SNAP ${ }_{c}$ does not reveal any stoichiometric component beside the three expected subunits. This mini-SNAP ${ }_{c}$ was still able to recruit TBP to the TATA box of an RNA Pol III snRNA promoter. We have shown previously that recruitment of TBP by $\mathrm{SNAP}_{\mathrm{c}}$ is dependent on the amino-terminal domain of TBP, and have speculated that it may occur through a direct protein-protein contact involving the amino-terminal domain of TBP and $\mathrm{SNAP}_{\mathrm{c}}$ (Mittal and Hernandez 1997). If this is the case, the target contacted by the amino-terminal domain of TBP must reside within miniSNAP $_{c}$.

Strikingly, mini-SNAP is capable of directing both basal RNA Pol II and Pol III snRNA gene transcription. Thus, just two and one-third subunits of $\mathrm{SNAP}_{\mathrm{c}}$ contain all of the required information for assembly of an RNA Pol II initiation complex, and together with TBP bound to the TATA box, an RNA Pol III initiation complex. Mini-SNAP ${ }_{c}$ was, however, consistently less efficient than $\mathrm{SNAP}_{\mathrm{c}}$ in directing basal RNA Pol II transcription, suggesting that it may be less efficient at recruiting RNA Pol II-specific transcription factors such as TFIIA and TFIIB, which are required for RNA polymerase II snRNA gene transcription (Kuhlman et al. 1999). But the main role of the $\mathrm{SNAP}_{\mathrm{c}}$ proteins not present in mini-SNAP $\mathrm{C}_{\mathrm{c}}$ is likely to be regulatory. The carboxy-terminal region of SNAP190 mediates activation by the Oct-1 POU domain; other $\mathrm{SNAP}_{\mathrm{c}}$ proteins may be involved in the response to the Oct-1 activation domains, which is not scored in our assays. And importantly, we used naked DNA templates for the in vitro transcription assays. Complete $\mathrm{SNAP}_{\mathrm{c}}$ may be required for transcription from chromatin templates.

The carboxy-terminal region of SNAP190

down-regulates binding of $S N A P_{c}$ to the PSE

$\mathrm{SNAP}_{\mathrm{c}}$ binds cooperatively with the Oct-1 POU domain to DNA, and this results in transcription activation (Murphy et al. 1992; Mittal et al. 1996; Ford et al. 1998). Thus, Oct-1 activates transcription through at least two mechanisms. One involves the Oct-1 POU domain, consists in recruitment of $\mathrm{SNAP}_{\mathrm{c}}$ through a direct proteinprotein contact (Ford et al. 1998), and is scored in vitro; the other involves the Oct-1 activation domains (Tanaka et al. 1992), functions through another mechanism than $\mathrm{SNAP}_{\mathrm{c}}$ recruitment to histone-free DNA (Ford and Hernandez 1997), and is not scored in our in vitro transcription system. We find that mini-SNAP ${ }_{c}$ binds more efficiently to the PSE than $\operatorname{SNAP}_{c}$, as well in fact as $\operatorname{SNAP}_{c}$ in the presence of Oct-1 POU. Accordingly, mini-SNAP directs levels of RNA Pol III snRNA gene transcription in vitro that correspond to the activated levels obtained with $\mathrm{SNAP}_{\mathrm{c}}$. This observation confirms that the Oct-1 POU domain activates transcription by recruitment of $\mathrm{SNAP}_{\mathrm{c}}$. It also reveals that $\mathrm{SNAP}_{\mathrm{c}}$ contains a built-in mechanism to down-regulate its binding to DNA, which is counteracted by a direct protein-protein contact with the Oct-1 POU domain.

The ability of SNAP to down-regulate its own binding is reminiscent of our previous observations with TBP (Mittal and Hernandez 1997). In this case, full-length human TBP binds much less efficiently to TATA boxes 
than a truncation missing the nonconserved amino-terminal domain of the protein. However, the TBP aminoterminal domain is absolutely required to promote cooperative binding with $\mathrm{SNAP}_{\mathrm{c}}$. Thus, as illustrated in Figure 9, the assembly of an RNA Pol III transcription initiation complex on an snRNA promoter probably involves at least two cooperative binding events, both of which relieve an inhibition of binding. We imagine that the amino-terminal domain of TBP and the carboxy-terminal domain of SNAP190 mask partially the DNAbinding domains of $\mathrm{TBP}$ and $\mathrm{SNAP}_{\mathrm{c}}$, respectively (top). On cooperative binding of TBP and $\mathrm{SNAP}_{\mathrm{c}}$, the aminoterminal domain of TBP, and in particular amino acids 1-96 including the run of Q residues (Mittal and Hernandez 1997), interacts with $\mathrm{SNAP}_{\mathrm{c}}$ in a manner independent of SNAP19, SNAP45, and the carboxy-terminal domain of SNAP190. Similarly, on cooperative binding of
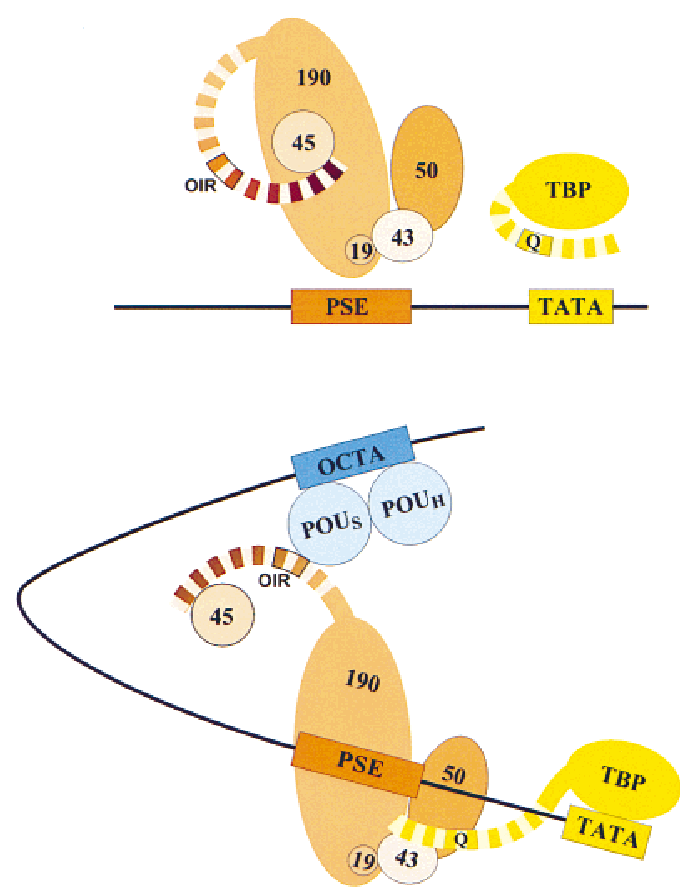

Figure 9. Cooperative binding of Oct-1 POU, $\mathrm{SNAP}_{\mathrm{c}}$ and TBP on an RNA Pol III snRNA promoter. (Top) $\mathrm{SNAP}_{\mathrm{c}}$ and TBP off the DNA. The binding of $\mathrm{SNAP}_{\mathrm{c}}$ to the PSE is down-regulated by the carboxy-terminal part of SNAP190 (striped ribbon), which may be partially masking the DNA-binding domain of the complex, and the binding of TBP to the TATA box is similarly down-regulated by the amino-terminal part of TBP. (Bottom) The factors bound together with Oct-1 POU to an RNA Pol III snRNA promoter. Oct-1 POU and $\mathrm{SNAP}_{\mathrm{c}}$ bind cooperatively to the promoter through interactions involving the Oct-1 $\mathrm{POU}_{\mathrm{S}}$ domain and the octamer-interacting region (OIR) between amino acids 869 and 912 within the carboxy-terminal part of the largest SNAP $_{\mathrm{c}}$ subunit, SNAP190 (Ford and Hernandez 1997; Mittal and Hernandez 1997; Ford et al. 1998). SNAP ${ }_{c}$ and TBP bind cooperatively through interactions involving the aminoterminal 505 amino acids of SNAP190, SNAP43, and SNAP50, or a subset of these $\mathrm{SNAP}_{\mathrm{c}}$ components, and amino acids 1-96 (which include the Q-rich region) of human TBP (Mittal and Hernandez 1997).
SNAP $_{c}$ and Oct-1 POU, the carboxy-terminal domain of SNAP190 and more precisely amino acids 869-912 (Ford

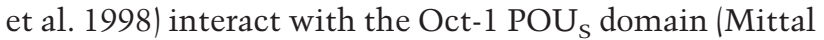
et al. 1996; Ford and Hernandez 1997) (bottom). Thus, for both factors, the part of the protein that down-regulates binding to DNA is also required for up-regulation of binding by cooperative interactions with transcription factor partners that recognize the same promoter, and thus, in effect, serves as a switch.

It is striking that both TBP and $\mathrm{SNAP}_{\mathrm{c}}$ have very slow dissociation and association rates (Hahn et al. 1989; Holdsworth et al. 1992; Imbalzano et al. 1994; Coleman and Pugh 1995; Taggart and Pugh 1996; Weideman et al. 1997; Hoopes et al. 1998). In the case of $\mathrm{SNAP}_{\mathrm{c}}, 50 \%$ of $\mathrm{SNAP}_{\mathrm{c}}-\mathrm{PSE}$ complexes are still present $60 \mathrm{~min}$ after challenge with a vast excess of unlabeled PSE competitor DNA, and cooperative binding with the Oct-1 POU domain accelerates the association of the complex with DNA with little effect on the dissociation rate (Mittal et al. 1996). In sharp contrast, the Oct-1 POU domain has very fast association and dissociation rates (W. Herr, pers. comm.). Thus, the Oct-1 POU domain could serve as a sensor identifying octamer-binding sites. In those cases in which the octamer sequence is within an snRNA promoter, that is, in the vicinity of a PSE, cooperative interactions would then recruit $\mathrm{SNAP}_{\mathrm{c}}$ and, in RNA Pol III snRNA promoters, TBP.

Other examples of basal transcription factors with built-in negative control of binding include E. coli $\sigma^{70}$ (Dombroski et al. 1992, 1993) and the largest TFIID subunit from both Drosophila $\left(\mathrm{dTAF}_{\mathrm{II}} 230\right)$ and yeast (yTAF $\left.{ }_{\text {II }} 145\right)$ (Kokubo et al. 1993, 1994, 1998; Bai et al. 1997). The first example is a case of autoinhibition, in which the amino-terminal region of $\sigma^{70}$ inhibits the binding of the carboxy-terminal domain of the protein to core promoter elements (Dombroski et al. 1992, 1993). In the second case, the amino-terminal region of the largest subunit of TFIID interacts directly with the DNA-binding subunit of TFIID, TBP, and inhibits its binding. This amino-terminal region competes with TFIIA for binding to $\mathrm{TBP}$, suggesting that it participates in a mechanism of transcription activation involving TFIIA (Kokubo et al. 1998). The mechanism by which the inhibition of binding is relieved is, however, not known. By homology with TBP and $\mathrm{SNAP}_{\mathrm{c}}$ in the snRNA promoters, we suspect that the amino-terminal domain of the largest TFIID subunit becomes engaged in cooperative binding interactions with another transcription factor binding to the same promoter, thus relieving the inhibition and instead increasing TFIID binding. Thus, a number of core promoter binding factors may be similar to $\mathrm{SNAP}_{\mathrm{c}}$ and TBP in possessing a mechanism that down-regulates their own binding and is reversed through protein-protein contacts with factors binding to the same promoter. Such a partner-activated switch probably serves to ensure that basal transcription factors, which often do not bind DNA with great sequence specificity (Coleman and Pugh 1995) and have strikingly slow off-rate, are targeted specifically to promoter sequences rather than to random cryptic sites present in the genome. 


\section{Materials and methods}

Expression of proteins in E. coli

The wild-type GST-Oct-1 POU and GST-SNAP45 proteins were expressed in E. coli BL21 (DE3) cells with the T7 expression system, as described before (Mittal et al. 1996). The proteins were purified by binding to glutathione-agarose beads and elution with thrombin, which removed the GST moiety of the fusion proteins. Protein purity was assessed by Coomassie staining of a $15 \%$ SDS-polyacrylamide gel.

\section{Assembly and purification of $S N A P_{c}$ and partial $S N A P_{c} S$}

SNAP $_{c}$ or partial SNAP ${ }_{c}$ s containing SNAP190 with a His tag at its carboxyl terminus and SNAP50 with an HA tag at its amino terminus were assembled and purified as described before (Henry et al. 1998b). Mini-SNAP ${ }_{c}$ was purified first over protein A-agarose beads (Boehringer Mannheim) covalently coupled to an anti-SNAP190 antibody (CS696). Bound proteins were eluted with a buffer containing $0.5 \mathrm{mg} / \mathrm{ml}$ of the peptide against which the antibody was raised in $20 \mathrm{~mm}$ HEPES (pH 7.9), $5 \mathrm{~mm} \mathrm{MgCl}_{2}$, $0.1 \%$ Tween $20,15 \%$ glycerol, $100 \mathrm{~mm} \mathrm{KCl}, 1 \mathrm{~mm}$ dithiothreitol (DTT), and the following protease inhibitors: $0.5 \mathrm{~mm}$ PMSF, 1 $\mathrm{mm}$ benzamidine, $2 \mu \mathrm{g} / \mathrm{ml}$ aprotonin, $1 \mu \mathrm{g} / \mathrm{ml}$ leupeptin, $1 \mathrm{~mm}$ sodium bisulfite, $0.5 \mu \mathrm{M}$ pepstatin $\mathrm{A}$, and $2 \mathrm{~mm}$ pefablock (Boehringer-Mannheim). Alternatively, mini-SNAP ${ }_{c}$ was purified first over nickel agarose beads as described previously for $\mathrm{SNAP}_{\mathrm{c}}$ (Henry et al. 1998b). In both cases, the eluates were further purified over protein $\mathrm{G}$-agarose beads coupled to the anti-HA mAb 12CA5 (Niman et al. 1983). The bound proteins were eluted with the same buffer as above containing $0.7 \mathrm{mg} / \mathrm{ml}$ HA peptide. The composition of the complexes was checked by immunoblots with the following antibodies: anti-SNAP19 (CS543, anti-p19Cpep; Henry et al. 1998b), anti-SNAP43 (CS49, anti-CSH375; Henry et al. 1995), anti-SNAP45 (CS234, $\alpha$-CSH467; Sadowski et al. 1996), anti-SNAP50 (CS303, $\alpha$-CSH482; Henry et al. 1996), anti-SNAP190 (CS402, Ab402;

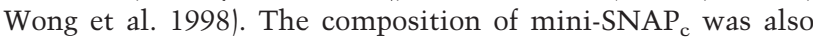
checked by SDS-PAGE followed by silver staining.

\section{EMSAs}

The EMSAs involving $\mathrm{SNAP}_{\mathrm{c}}$ or $\mathrm{SNAP}_{\mathrm{c}}$ and Oct-1 POU were performed as described (Mittal et al. 1996), and those involving SNAP $_{c}$ and TBP were performed as described (Mittal and Hernandez 1997). The antibodies used in Figure 5B were as follows: anti-SNAP190 N-ter (CS696), anti-SNAP190 C-ter (CS402, Ab402; Wong et al. 1998), anti-SNAP50 (CS303, a $\alpha$-CSH482; Henry et al. 1996), anti-SNAP45 (CS234, $\alpha$-CSH467; Sadowski et al. 1996), anti-SNAP43 (CS49, anti-CSH375; Henry et al. 1995); anti-SNAP19 (CS543, anti-p19Cpep; Henry et al. 1998b).

\section{DNase I footprinting}

The probes for DNase I footprinting were prepared by PCR with two primers, one of which was $5^{\prime}$ end-labeled with $\left[\gamma^{-32} \mathrm{P}\right]$ ATP. The binding reaction was performed at $30^{\circ} \mathrm{C}$ for $30 \mathrm{~min}$ in a total volume of $50 \mu \mathrm{l}$ and contained $20 \mathrm{~mm}$ HEPES (pH 7.9), 100 mм KCl, $5 \mathrm{~mm} \mathrm{MgCl}_{2}, 0.2$ mм EDTA, 10\% glycerol, 1 mм DTT, $0.2 \mu \mathrm{g}$ each of poly[d(G-C)-(G-C)] and pUC118, $2 \%$ polyvinyl alcohol, and 10,000 cpm of radiolabeled probe. DNase I digestion was carried out as described (Schmidt et al. 1989), and the reaction products were analyzed on a $7 \%$ polyacrylamide-urea gel.

\section{In vitro transcription}

The U1 and U6 constructs used in Figure 7 have been described in Sadowski et al. (1993), and the reporter U6 construct used in Figure 8B has been described in Mittal et al. (1996). HeLa cell extracts were depleted of $\mathrm{SNAP}_{c}$ with an equal volume of a mixture of protein A-agarose beads cross-linked to antiSNAP19 (CS543, anti-p19Cpep; Henry et al. 1998b), antiSNAP45 (CS234, $\alpha$-CSH467; Sadowski et al. 1996), and antiSNAP190 (CS696) antibodies. Control extracts were depleted similarly, but with beads cross-linked to preimmune antibodies. The transcription reactions were performed as described before (Sadowski et al. 1993).

\section{Acknowledgments}

We thank W.P. Tansey for comments on the manuscript, W. Herr for discussion, S. Sepehri for a constant supply of U6 RNA probe and HeLa cell extract, and M. Ockler, J. Duffy, and P. Renna for artwork and photography. This work was funded in part by National Institutes of Health grant GM38810. We are supported by the Howard Hughes Medical Institute.

The publication costs of this article were defrayed in part by payment of page charges. This article must therefore be hereby marked 'advertisement' in accordance with 18 USC section 1734 solely to indicate this fact.

\section{References}

Bai, Y., G.M. Perez, J.M. Beechem and P.A. Weil. 1997. Structure-function analysis of TAF130: Identification and characterization of a high-affinity TATA-binding protein interaction domain in the $\mathrm{N}$ terminus of yeast TAF(II)130. Mol. Cell. Biol. 17: 3081-3093.

Chen, J.-L., L.D. Attardi, P.C. Vrreijzer, K. Yokomori, and R. Tjian. 1994. Assembly of recombinant TFIID reveals differential coactivator requirements for distinct transcriptional activators. Cell 79: 93-105.

Coleman, R.A. and B.F. Pugh. 1995. Evidence for functional binding and stable sliding of the TATA binding protein on nonspecific DNA. J. Biol. Chem. 270: 13850-13859.

Dombroski, A.J., W.A. Walter, M.T.J. Record, D.A. Siegele, and C.A. Gross. 1992. Polypeptides containing highyl conserved regions of transcription initiation factor $\sigma^{70}$ exhibit specificity of binding to promoter DNA. Cell 70: 501-512.

Dombroski, A.J., W.A. Walter, and C.A. Gross. 1993. Aminoterminal amino acids modulate $\sigma$-factor DNA-binding activity. Genes \& Dev. 7: 2446-2455.

Ford, E. and N. Hernandez. 1997. Characterization of a trimeric complex containing Oct-1, $\mathrm{SNAP}_{\mathrm{c}}$, and DNA. J. Biol. Chem. 272: $16048-16055$.

Ford, E., M. Strubin, and N. Hernandez. 1998. The Oct-1 POU domain activates snRNA gene transcription by contacting a region in the $\mathrm{SNAP}_{\mathrm{c}}$ largest subunit that bears sequence similarities with the Oct-1 coactivator OBF-1. Genes \& Dev. 12: 3528-3540.

Hahn, S., S. Buratowski, P.A. Sharp, and L. Guarente. 1989. Yeast TATA-binding protein TFIID binds to TATA elements with both consensus and nonconsensus DNA sequences. Proc. Natl. Acad. Sci. 86: 5718-5722.

Henry, R.W., C.L. Sadowski, R. Kobayashi, and N. Hernandez. 1995. A TBP-TAF complex required for transcription of human snRNA genes by RNA polymerases II and III. Nature 374: 653-657.

Henry, R.W., B. Ma, C.L. Sadowski, R. Kobayashi, and N. Hernandez. 1996. Cloning and characterization of SNAP50, a subunit of the snRNA activating protein complex $\mathrm{SNAP}_{\mathrm{c}}$. 
EMBO J 15: 7129-7136.

Henry, R.W., E. Ford, R. Mital, V. Mittal, and N. Hernandez. 1998a. Crossing the line between RNA polymerases: Transcription of human snRNA genes by RNA polymerases II and III. Cold Spring Harbor Symp. Quant. Biol.Volume 63, pp. 111-120.

Henry, R.W., V. Mittal, B. Ma, R. Kobayashi and N. Hernandez. 1998b. Assembly of a functional, core promoter complex $\left(\mathrm{SNAP}_{\mathrm{c}}\right)$ shared by RNA polymerase II and III. Genes \& Dev. 12: 2664-2672.

Herr, W., R.A. Sturm, R.G. Clerc, L.M. Corcoran, D. Baltimore, P.A. Sharp, H.A. Ingraham, M.G. Rosenfeld, M. Finney, G. Ruvkun, and H.R. Horvitz. 1988. The POU domain: A large conserved region in the mammalian pit-1, oct-1, oct-2, and Caenorhabditis elegans unc-86 gene products. Genes \& Dev. 2: 1513-1516.

Holdsworth, M.J., C. Grierson, W. Schuch, and M. Bevan. 1992. DNA-binding properties of cloned TATA-binding protein from potato tubers. Plant Mol. Biol. 19: 455-464.

Hoopes, B.C., J.F. LeBlanc, and D.K. Hawley. 1998. Contributions of the TATA box sequence to rate-limiting steps in transcription initiation by RNA polymerase II. J. Mol. Biol. 277: 1015-1031.

Imbalzano, A., K.S. Zaret, and R.E. Kingston. 1994. Transcription factor (TF) IIB and TFIIA can independently increase the affinity of the TATA-binding protein for DNA. I. Biol. Chem. 269: 8280-8286.

Kokubo, T., D.-W. Gong, S. Yamashita, M. Horikoshi, R.G. Roeder, and Y. Nakatani. 1993. Drosophila 230-kD TFIID subunit, a functional homolog of the human cell cycle gene product, negatively regulates DNA binding of the TATA box-binding subunit of TFIID. Genes \& Dev. 7: 1033-1046.

Kokubo, T., S. Yamashita, M. Horikoshi, R.G. Roeder, and Y. Nakatani. 1994. Interaction between the N-terminal domain of the $230-\mathrm{kDa}$ subunit and the TATA box-binding subunit of TFIID negatively regulates TATA-box binding. Proc. Natl. Acad. Sci. 91: 3520-3524.

Kokubo, T., M.J. Swanson, J.I. Nishikawa, A.G. Hinnebusch, and Y. Nakatani. 1998. The yeast TAF145 inhibitory domain and TFIIA competitively bind to TATA- binding protein. Mol. Cell. Biol. 18: 1003-1012.

Kuhlman, T.C., H. Cho, D. Reinberg, and N. Hernandez. 1999. The general transcription factors IIA, IIB, IIF, and IIE are required for RNA polymerase II transcription from the human U1 snRNA promoter. Mol. Cell. Biol. 19: 2130-2141.

Lobo, S.M. and N. Hernandez. 1994. Transcription of snRNA genes by RNA polymerases II and III. Transcription, mechanisms and regulation (ed. R.C. Conaway and J.W. Conaway), pp. 127-159. Raven Press, New York, NY.

Luscher, B. and R.N. Eisenman. 1990. New light on Myc and Myb. Part II. Myb. Genes \& Dev. 4: 2235-2241.

Mittal, V. and N. Hernandez. 1997. Role for the amino-terminal region of human TBP in U6 snRNA transcription. Science 275: 1136-1140.

Mittal, V., M.A. Cleary, W. Herr, and N. Hernandez. 1996. The Oct-1 POU-specific domain can stimulate small nuclear RNA gene transcription by stabilizing the basal transcrip-

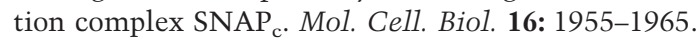

Murphy, S., J.-B. Yoon, T. Gerster, and R.G. Roeder. 1992. Oct-1 and Oct-2 potentiate functional interactions of a transcription factor with the proximal sequence element of small nuclear RNA genes. Mol. Cell. Biol. 12: 3247-3261.

Niman, H.L., R.A. Houghten, L.E. Walker, R.A. Reisfeld, I.A. Wilson, J.M. Hogle, and R.A. Lerner. 1983. Generation of protein-reactive antibodies by short peptides is an event of high frequency: Implications for the structural basis of im- mune recognition. Proc. Natl. Acad. Sci. 80: 4949-4953.

Nomura, N., M. Takahashi, M. Matsui, S. Ishii, T. Date, S. Sasamoto, and R. Ishizaki. 1988. Isolation of human cDNA clones of myb-related genes, A-myb and B-myb. Nucleic Acids Res. 16: 11075-11089.

Sadowski, C.L., R.W. Henry, S.M. Lobo, and N. Hernandez. 1993. Targeting TBP to a non-TATA box cis-regulatory element: A TBP-containing complex activates transcription from snRNA promoters through the PSE. Genes \& Dev. 7: 1535-1548.

Sadowski, C.L., R.W. Henry, R. Kobayashi, and N. Hernandez. 1996. The SNAP45 subunit of the small nuclear RNA (snRNA) activating protein complex is required for RNA polymerase II and III snRNA gene transcription and interacts with the TATA box binding protein. Proc. Natl. Acad. Sci. 93: 4289-4293.

Schmidt, M.C., Q. Zhou, and A.J. Berk. 1989. Sp1 activates transcription without enhancing DNA-binding activity of the TATA box factor. Mol. Cell. Biol. 8: 3299-3307.

Taggart, A.K. and B.F. Pugh. 1996. Dimerization of TFIID when not bound to DNA. Science 272: 1331-1333.

Tanaka, M., J.-S. Lai, and W. Herr. 1992. Promoter-selective activation domains in Oct-1 and Oct- 2 direct differential activation of an snRNA and mRNA promoter. Cell 68: 755767.

Tansey, W.P. and W. Herr. 1997. TAFs: Guilt by association? Cell 88: $729-732$.

Weideman, C.A., R.C. Netter, L.R. Benjamin, J.J. McAllister, L.A. Schmiedekamp, R.A. Coleman, and B.F. Pugh. 1997. Dynamic interplay of TFIIA, TBP and TATA DNA. J. Mol. Biol. 271: 61-75.

Wong, M.W., R.W. Henry, B. Ma, R. Kobayashi, N. Klages, P. Matthias, M. Strubin, and N. Hernandez. 1998. The large subunit of basal transcription factor $\mathrm{SNAP}_{\mathrm{c}}$ is a Myb domain protein that interacts with Oct-1. Mol. Cell. Biol. 18: 368377. 


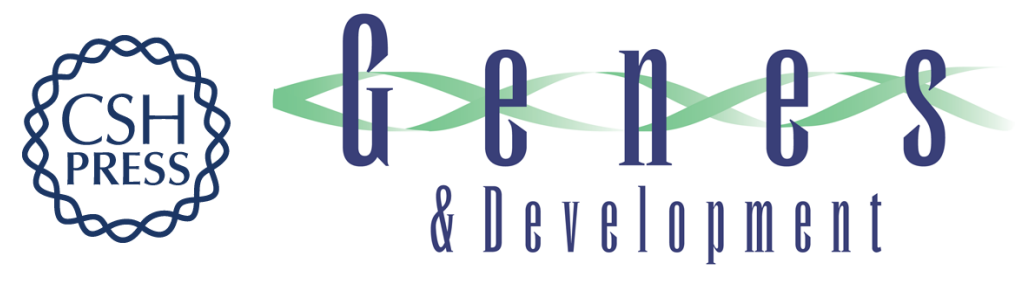

\section{SNAP $_{c}$ : a core promoter factor with a built-in DNA-binding damper that is deactivated by the Oct-1 POU domain}

Vivek Mittal, Beicong Ma and Nouria Hernandez

Genes Dev. 1999, 13:

References This article cites 33 articles, 21 of which can be accessed free at:

http://genesdev.cshlp.org/content/13/14/1807.full.html\#ref-list-1

License

Email Alerting

Receive free email alerts when new articles cite this article - sign up in the box at the top

Service right corner of the article or click here.

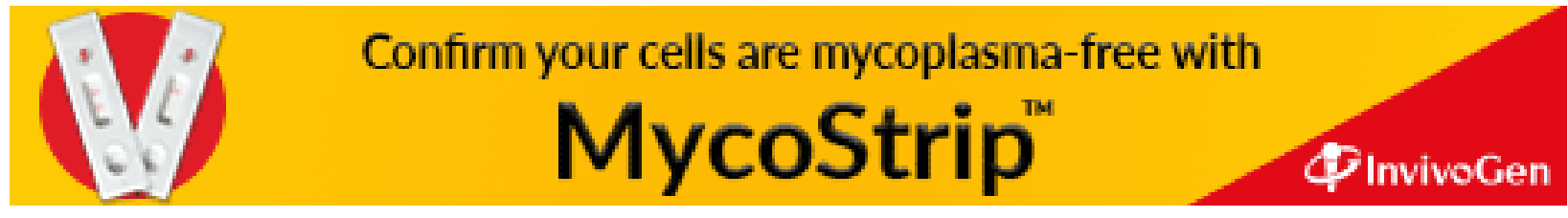

